International Journal of Bifurcation and Chaos, Vol. 11, No. 8 (2001) 2145-2162

(c) World Scientific Publishing Company

\title{
COMBINATORIAL CONTROL OF GLOBAL DYNAMICS IN A CHAOTIC DIFFERENTIAL EQUATION
}

\author{
ERIK M. BOLLT* \\ Department of Mathematics, 572 Holloway Rd., U.S. Naval Academy, \\ Annapolis, MD 21402-5002, USA
}

Received September 6, 2000; Revised January 9, 2001

\begin{abstract}
Controlling chaos has been an extremely active area of research in applied dynamical systems, following the introduction of the Ott, Grebogi, Yorke (OGY) technique in 1990 [Ott et al., 1990], but most of this research based on parametric feedback control uses local techniques. Associated with a dynamical system which pushes forward initial conditions in time, transfer operators, including the Frobenius-Perron operator, are associated dynamical systems which push forward ensemble distributions of initial conditions. In [Bollt, 2000a, 2000b; Bollt \& Kostelich, 1998], we have shown that such global representations of a discrete dynamical system are useful in controlling certain aspects of a chaotic dynamical system which could only be accessible through such a global representation. Such aspects include invariant measure targeting, as well as orbit targeting. In this paper, we develop techniques to show that our previously discrete time techniques are accessible also to a differential equation. We focus on the Duffing oscillator as an example. We also show that a recent extension of our techniques by Góra and Boyarsky [1999] can be further simplified and represented in a convenient and compact way by using a tensor product.
\end{abstract}

\section{Introduction}

The purpose of this work is to demonstrate parametric feedback control over global aspects of a chaotic differential equation. Specifically, we choose the popular Duffing oscillator [Alligood et al., 1996] as our main example. We have shown in our previous work [Bollt, 2000a, 2000b; Bollt \& Kostelich, 1998] that the global action of a discrete dynamical system is well approximated by a transfer matrix, and that this fact is useful for designing global control strategies. The purpose of this paper is to review these techniques in a unifying framework, and then to explicitly show for the first time here, that the $\varepsilon$-chain pseudo-orbits implied by walks through a directed graph representation can be realized and stabilized by appropriately designed parameter feedback control, specifically in the case of a differential equation on Poincaré surface of section.

In [Bollt \& Kostelich, 1998], we used a discretization of a global transfer operator on a grid, which is hence a transfer matrix $[A]_{i, j}$. Given this approximation, we showed that standard path searching algorithms [Gould, 1988; Bondy \& Murty, 1976], such as the BFS or Dijkstra's algorithms, can be used to target optimal walks through the graph, which by construction, are fast $\varepsilon$-chain pseudoorbits of the dynamical system. In [Bollt, 2000a], we used another sort of transfer operator representation of the dynamical system; Ulam's method [Ulam, 1960] again uses an arbitrary grid, but to

*E-mail: bollt@nadn.navy.mil

http://mathweb.mathsci.usna.edu/faculty/bolltem/ 
approximate the Frobenius-Perron operator $P_{f}$, by a probability matrix $[S]_{i, j}$. We showed that careful manipulation of the entries of this matrix can be performed to design a desirable dominant eigenvector, and then by the so-called Inverse-Ulam problem (IUP) [Bollt, 2000a], a nearby dynamical system can be constructed which has exactly the redesigned $\mathrm{F}-\mathrm{P}$ operator, and hence exactly the desired invariant density. The IUP constructs a nearby map, which we previously described as implying an openloop (no feedback) control strategy. On the other hand we now interpret such a solution as giving an $\varepsilon$-chain pseudo-orbit of the original map.

In this paper, we design techniques to formulate global control strategies by combinatorial methods, and then to realize these rough pseudo-orbits found by combinatorial methods as true orbits of a differential equation by means of multiparametric feedback-control. We assume a discrete dynamical system, $f_{\lambda}: M \rightarrow M, M \in \Re^{n}$, where generally in this paper, this map is derived from a set of ordinary differential equations by Poincaré surface mapping. For such a map, one can ask what is the long term statistics of ensembles of initial conditions under $f_{\lambda}$. We take $\lambda \in \Re^{m}$ to be the set of adjustable control parameters. We will show control of statistical aspects of such systems, as well as global targeting.

Ott, Grebogi, and Yorke, introduced the OGY method [Ott et al., 1990], to stabilize an unstable periodic orbit embedded in a chaotic attractor. This original idea opened the field of controlling chaos. During the last decade, a major emphasis of dynamical systems theory research has been focused on the realization that sensitive dependence provides that control of a chaotic dynamical system can be flexible and efficient. We cannot begin to describe the scope of literature in this active area of research, or its many important and physical applications, and so we refer the reader to recent books [Ott et al., 1994; Chen \& Dong, 1998]. A main idea in this literature is that the sensitive dependence, characteristic of chaos, is actually advantageous to build a highly agile control system in which a small deliberate perturbation can cause a large response. A chaotic attractor can be considered as an unlimited reservoir of widely varied behaviors. OGY [Ott et al., 1990] uses a local linear feedback control loop by targeting the stable manifold of an unstable fixed point through small parameter variations. Ergodicity causes an arbitrary initial condition to eventually wander close enough to the fixed point that the tiny parameter variations are sufficient to stabilize the orbit. With this in mind, it can be said that the global strategy of OGY is patience plus ergodicity. The "butterfly effect" allows us to quickly steer trajectories to targets with only small perturbations, [Shinbrot et al., 1990; Shinbrot et al., 1992; Kostelich et al., 1993]; this is called "targeting," and is related to the time-optimal control problem for maps [Bollt \& Meiss, 1995]. However, the majority of research has relied on local analysis, which has limited application for global control objectives. The above describes feedback control of single trajectories, and the techniques are generally local in nature. The present work is distinguished due to the global scope of our techniques, in that we work with approximations of the transfer operator representations of the action of a dynamical system on ensembles of initial conditions, rather than directly with the dynamical system which operates on single initial conditions. We define more general control objectives to include controlling, or avoiding (anticontrolling [Schiff et al., 1994; Chen \& Lai, 1997]), long term statistical behavior over an attractor. We call this targeting of invariant measures.

While recently there have been other solutions to Inverse Frobenius-Perron problems [Góra \& Boyarsky, 1993, 1996, 1997, 1998, 1999; Koga, 1991; Baranovsky \& Daems, 1995; Pingel et al., 1999], these usually require large variations in the map. Hence, those IFPP strategies pertain only to maps. Our formulation of the problem is unique, in that we allow both for small changes to the map, and general target densities. The approach here can be considered as a perturbation result relative to a known dynamical system, and therefore can be realized in a flow, by appropriately small parameter variations, relative to a known experimental/numerical dynamical system. This follows an assumption that small next response variations on the surface of section are continuous with respect to parameter variations.

The layout of this paper is as follows. In Sec. 2 we introduce the Duffing oscillator as our standard example, including the Duffing map and standard parameter values chosen, and we describe our control objectives and goals. In Sec. 3, we give some background on transfer operators associated with a point transformation dynamical system. In Sec. 4, we discuss using graph theoretic algorithms to find optimal paths through the graph approximations of the transfer operators, and their relation to paths of the dynamical system. In Sec. 5, we review [Bollt, 2000a] our solution of the IFPP for completeness, using notation recently introduced by Gora and 
Boyarsky [1999], but then we introduce a newer and simpler representation of this formalism. In Sec. 6 , we discuss how an $\varepsilon$-chain pseudo-orbit is implied by a path through a graph approximation of the transfer operator, and how these rough orbits may be closed to become true orbits of a differential equation by using parameteric feedback control. In Sec. 7, we give numerical examples both of targeting fast trajectories, as well as targeting invariant densities, in the case of the Duffing oscillator. Concluding remarks are found in Sec. 8.

\section{Goal and Example Model}

In this paper, we will demostrate control over global aspects of a differential equation by parametric feedback variations control. We demonstrate that the $\varepsilon$-chain pseudo-orbits constructed using a graph approximation of the dynamical system's global action on its phase space, which achieve various global objectives, can be stabilized to represent a true orbit of a differential equation, by multiparameteric feedback control. Furthermore the graph approximation of the global action is easily learned by observing a single chaotically wandering orbit through the partition.

As an example of dynamical system, we choose the popular Duffing oscillator [Alligood et al., 1996], written in autonomous form,

$$
\begin{aligned}
& x^{\prime}=y \\
& y^{\prime}=-a y-x^{3}+x+b \sin (\tau), \\
& \tau^{\prime}=1
\end{aligned}
$$

with the nominal parameter values $\lambda_{0}=\left(a_{0}, b_{0}\right)=$ $(0.02,3)$. A Poincaré map is found by stroboscopic section, every time $\tau$ pierces a multiple of $2 \pi$. See Fig. 1.

We will demonstrate two different kinds of control over global aspects of this dynamical system.

1. Generalized Targeting of "short paths" from an initial point $\mathbf{z}_{a}$ to a target point $\mathbf{z}_{b}$. By "generalized targeting," we mean that the word "short" need not simply weigh each iterate equally. The other positive weight is associated with transitions from various regions of the phase space. See Sec. 4.

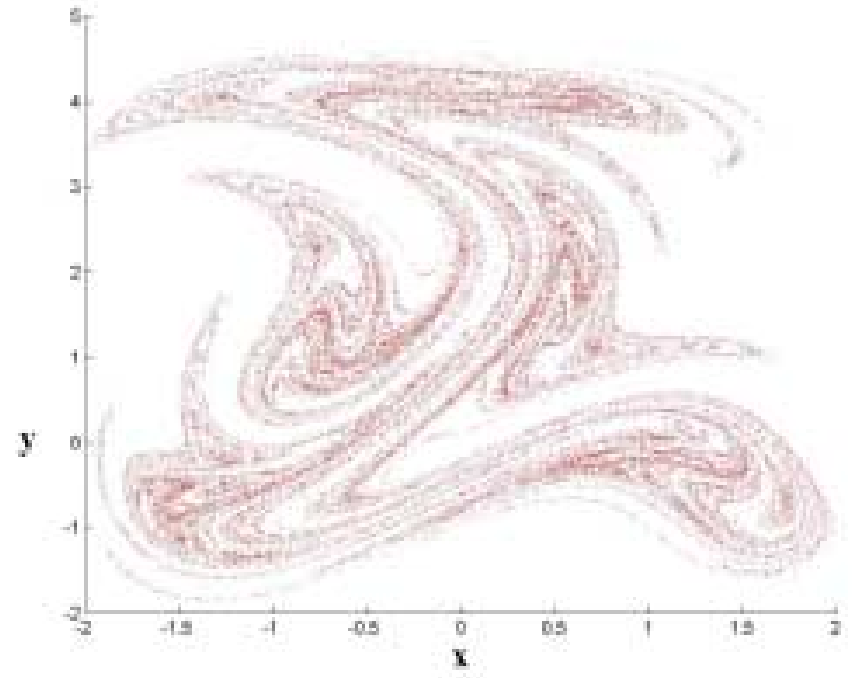

Fig. 1. Stroboscopic-2 $\pi$ map of Duffing oscillator: $x^{\prime \prime}+a x^{\prime}+$ $x^{3}-x=b \sin (t)$, where $\lambda_{0}=\left(a_{0}, b_{0}\right)=(0.02,3)$.

2. Alteration of Long Term Density Properties of the Attractor. By applying the Inverse Frobenius-Perron Problem, we will demonstrate the ability to choose an invariant density on the surface of section. Possible target densities include densities which:

(a) avoid unfavorable regions of the phase space (so-called anticontrol [Schiff et al., 1994; Chen \& Lai, 1997]),

(b) stabilize atomic densities supported over periodic orbits (globally stabilize periodic orbits),

(c) slightly varying densities from the natural density.

See Sec. 6.

The algorithms to achieve Objective 1 [Bollt \& Kostelich, 1998] and Objective 2 [Bollt, 2000a, $2000 \mathrm{~b}$ ] are related in that we describe the global action of a discrete map by either an unweighted or arbitrarily weighted directed graph (Objective 1) or a probability directed graph (Objective 2). The common thread is that walks through the graph correspond to $\varepsilon$-chain pseudo-orbits ${ }^{1}$ of the discrete map. This follows the construction of the graph: given a partition covering $\mathcal{G}_{n}=\left\{G_{i}\right\}_{i=1}^{n}$ of the attractor by $\varepsilon \times \varepsilon \times \cdots \times \varepsilon$ boxes $G_{i}$, then a single chaotically wandering "test" orbit $\left\{z_{k}\right\}_{k=0}^{N}$ can be used to "wire" the graph by recording a transition

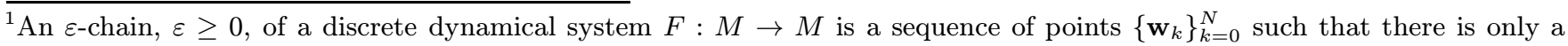
small normed error at each iterate: $\left\|\mathbf{w}_{k+1}-\mathbf{F}\left(\mathbf{w}_{k}\right)\right\| \leq \varepsilon$. Obviously, a true orbit of $F$ is an $\varepsilon$-chain in which $\varepsilon=0$.
} 
from vertex $i$ to vertex $j$ for each $\left(z_{k}, z_{k+1}\right)$ pair such that $z_{k} \in G_{i}$ and $z_{k+1} \in G_{j}$.

\section{Approximating Transfer Operators: Global Action of the Map}

Our global strategies were based, in [Bollt \& Kostelich, 1998] on discretizing the transfer operator $\mathcal{L}$, and in [Bollt, 2000a] on discretizing the closely related Frobenius-Perron operator $P_{f}(x)$. In this section, we give a short discussion of infinitedimensional transfer operators, and their approximation by transfer matrices on a fine $n$-element grid $\mathcal{G}_{n}=\left\{G_{i}\right\}_{i=1}^{n}$. In particular, we intend to point out the similiarity between a probability transfer matrix $[S]_{i, j}$ and $P_{f}(x)$, and other transfer matrices $[T]_{i, j}$.

Consider a discrete dynamical system as a map which advances initial conditions, $x \mapsto f(x) \mapsto$ $f^{2}(x) \mapsto \cdots$, which may be a Poincaré return map. The action of the map, on ensembles of initial conditions is addressed by integrating a transfer kernal [Cvitanovic, 1991],

$$
\mathcal{T}(y, x)=\delta(y-f(x)) .
$$

The Frobenius-Perron operator gives a linear dynamical system, $P_{f}: L^{1}(M) \rightarrow L^{1}(M)$, associated with the map $f$, which describes evolution of probability densities of initial conditions, $\rho(x) \in L^{1}(M)$, by integrating against the transfer kernal,

$$
\begin{aligned}
\rho_{n+1}(x) & =P_{f}\left[\rho_{n}(x)\right] \\
& =\int_{M} \delta(y-f(x)) \rho_{n}(y) d y \\
& =\sum_{\{y: f(y)=x\}} \frac{\rho_{n}(y)}{\left|f^{\prime}(y)\right|},
\end{aligned}
$$

where in multidimensions, $x \in \Re^{n},\left|f^{\prime}(y)\right|$ denotes the determinant of the Jacobian derivative [Lasota \& Mackey, 1997]. Ulam's method [Ulam, 1960] essentially projects this infinite dimensional operator onto a finite dimensional operator $[\mathrm{Li}, 1976]$ described by a matrix $[S]_{i, j}$ over a finite dimensional linear subspace of $L^{1}(M)$, generated by characteristic functions $\left\{\chi_{G_{i}}\right\}$ and supported over a uniform fine grid, $\mathcal{G}_{n}=\left\{G_{i}\right\}$. For a small grid square $G_{i}$, a continuous map $f$ is almost constant, which motivates Ulam's conjecture to approximate $P_{f}$ by the probability matrix,

$$
\begin{aligned}
P_{f}\left[\chi_{G_{i}}(x)\right] & \approx \sum_{i} S_{i, j} \chi_{\left[G_{i} \cap f^{-1}\left(G_{j}\right)\right]}(x) \\
& =\sum_{i} S_{i, j} \chi_{G_{i}}(x),
\end{aligned}
$$

where

$$
[S]_{i, j}=\frac{m\left(G_{i} \cap f^{-1}\left(G_{j}\right)\right)}{m\left(G_{i}\right)} .
$$

"Approximate" here means that as the grid $\mathcal{G}_{n}$ becomes finer, then the limit of the matrices $S_{n \times n}$ have dominant eigenvectors $v_{n}$ which converge weakly to the density of the invariant measure of $f$. To show sufficient conditions for the validity of Ulam's method is a difficult problem in functional analysis. ${ }^{2}$ We are not concerned here as to whether our initial transformation $f$ satisfies such assumptions, as we wish to control to a new transformation. In [Bollt, 2000a], we showed that a piecewise affine transformation can be constructed, which we call the Inverse Ulam Problem (IUP), such that $\mathcal{G}_{n}$ is a Markov partition for that modified transformation. Equation (5) gives exactly this new transformation's F-P operator. We use Eq. (5) only to get a rough snapshot of $P_{f}$, and it can be easily approximated by a Monte-Carlo approach, by considering the transitions of a long chaotic test orbit of $f$. Given $\left\{x_{i}\right\}_{i=0}^{N}$ and a partition $\mathcal{G}_{n}$, then let

$$
[S]_{i, j}=\frac{\# x_{k} \in G_{i} \text { such that } x_{k+1} \in G_{j}}{\# x_{k} \in G_{i}} .
$$

If we are interested in topological but not statistical properties, then we consider a "pure" transfer

\footnotetext{
${ }^{2}$ The Ulam conjecture was first proved by $\mathrm{Li}[1976]$ for 1-D transformations, and using bounded variation arguments requiring that $f \in$ piecewise $C^{2}[0,1]$, with $M>\inf \left|f^{\prime}\right|>2$. The $n$-dimensional generalization was proved by Froyland in the cases that $Q$ is a Markov partition of an expanding Anosov diffeomorphism, using symbol dynamics techniques in [Froyland, 1995], and by mixing arguments in [Froyland, 1998]. The conjecture was also proved [Boyarsky \& Lou, 1991], for expanding Jablonski transformations ( $n$-dimensional maps such that, on a grid, each componentwise function of $f$ is only a 1 -D function: $f_{Q_{i}}\left(x_{1}, \ldots, x_{n}\right)=\left(f_{Q_{i}}^{1}\left(x_{1}\right), f_{Q_{i}}^{2}\left(x_{2}\right), \ldots, f_{Q_{i}}^{n}\left(x_{n}\right)\right)$, using bounded-variation arguments modeled after the methods in [Li, 1976]. Likewise the methods in [Ding \& Zhou, 1994] for piecewise affine functions also use bounded variation arguments and therefore require an expanding transformation [Proppe et al., 1990].
} 

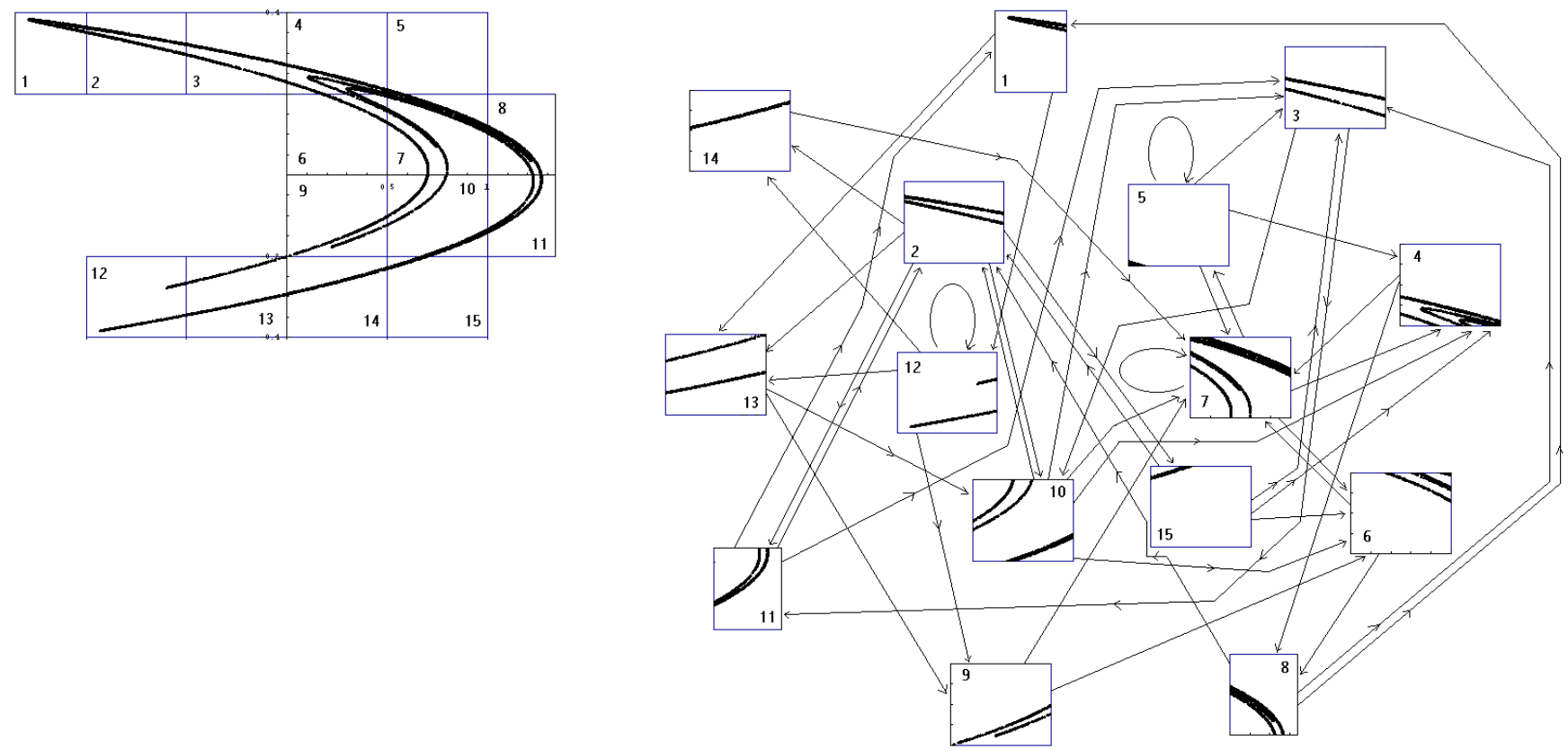

Fig. 2. Coarse-grained diGraph approximation of the action of Henon map. Representing a probability matrix $[S]_{i, j}$, edges carry probabilities of transitions, while representing a purely transfer matrix $[T]_{i, j}$, edges carry purely the nonzero entries of the matrix.

operator $\mathcal{L}$, which moves ensembles of initial conditions around, without regard to quantity. Ignoring rates, ${ }^{3}$ gives a transfer operator describing the purely one-step action of the map on the partition,

$$
\begin{aligned}
{[T]_{i, j} } & =\mathcal{L}\left(G_{i}, G_{j}\right) \\
& =\operatorname{floor}\left(\chi_{G_{j}} \circ P_{f}\left[\chi_{G_{i}}(x)\right]+1\right)-1 \\
& \equiv \operatorname{if}\left(G_{i} \cap f^{-1}\left(G_{j}\right)=\emptyset, 0,1\right) .
\end{aligned}
$$

This may arguably be an awkward way to determine whether or not there exists a point $x_{k} \in G_{i}$ such that $x_{k+1} \in G_{j}$. Our intent here is only to draw analogy to the formalism of the previous paragraph. In other words, $[S]_{i, j}>0 \Rightarrow[T]_{i, j}=1$, and $[S]_{i, j}=0 \Leftrightarrow[T]_{i, j}=0$, which is most easily determined by observing a test orbit.

Remark. Note that an approximation of a transfer matrix can be graphically represented by a directed graph, the idea of which is caricatured by the very coarse grid picture in Fig. 2. Our main point in this section is that the graph roughly approximates the global one-step action of the map, in the sense that any path through the graph has a realization as an $\varepsilon$-chain pseudo-orbit of the true map $f$. In other words, for each $i \rightarrow j$, there exists an $x \in G_{i}$ such that $f(x) \in G_{j}$, but this does not mean that such can be generalized to a two-step orbit. In short, the general partition $\mathcal{G}_{n}$ is not expected to be Markov [Bowen, 1975], and so a two-step path through the graph cannot be guaranteed to correspond to a real orbit of $f$, but only an $\varepsilon$-chain. If such were true, then the partition would generate the symbolic dynamics. This caveate is important for our application, since it means that we expect to use parameter variations at each step to force the controlled trajectory to follow an $\varepsilon$-chain with either desirable statistics or trajectory.

\section{Optimal Paths}

Optimal path searching is a classical problem in graph theory [Gould, 1988; Bondy \& Murty, 1976].

\footnotetext{
${ }^{3}$ Rates and approximations are considered by inspecting periodic orbits, and traces of transfer operators in the "cycling-theory" which provides that integrating the transfer kernal, Eq. (2) over a partition box $G_{i}, \int_{G_{i}} \delta(y-f(x)) d y \equiv P_{f}\left[\chi_{G_{i}}(x)\right]$, can be used to calculate the escape rate from $G_{i}$ [Kadanoff \& Tang, 1984], by considering $P_{f}^{n}\left[\chi_{G_{i}}(x)\right]$, most efficiently by the spectrum derived by zero's of the Fredholm determinant [Cvitanovic, 1988, 1991; Artuso et al., 1990] considering traces of iterates of this transfer operator $\operatorname{tr} \mathcal{T}^{n}$, which in turn has been described as a series expansion on periodic orbits.
} 
We recount here solutions for two different graph problems which pertain to our control problem.

\subsection{Iterate optimal}

The Breadth-First-Search (BFS) algorithm finds the shortest path from vertex $A$ (corresponding to the box containing the initial condition) to vertex $B$ in an unweighted directed graph [Gould, 1988; Bondy \& Murty, 1976]. The algorithm is as follows. Starting from vertex $A$, we inspect each adjacent vertex, those vertices that can be reached in one step. From these vertices, we check all adjacent vertices that have not yet been visited; these vertices can be reached from $A$ in exactly two steps. We continue until we find the target vertex among the vertices reachable in exactly $k$ steps.

To implement the algorithm, we maintain two lists. The second list contains the vertices that have never been visited (initially, all vertices except $A$ ), and the first list contains the vertices that have been reached in $k$ or fewer steps (initially, only vertex $A$ ). When a vertex is added to the first list, it is removed from the second list. Hence, the number of steps through the graph is bounded by the number of vertices, $n$. Starting at $A$, the program can find the distance (number of steps) to every vertex in the graph in time complexity $O(E)$, where $E$ is the number of edges in the graph. The output of BFS is a $3 \times n$ array, where the first entry is an identifier for the vertex, the second entry is the number of steps to reach the vertex, and the third entry identifies the predecessor of the vertex. Given the graph's connectivity (set of edges), we can easily backtrack through this array to find the shortest path from vertex $A$ to vertex $B$.

Figure 3 shows the results of an example application of BFS to the Duffing attractor. The color used for each box indicates the minimal number of iterates required to reach, starting from the initial box, marked by an "X", for an optimal $\varepsilon$-chain trajectory which will be stabilized by the techniques of Sec. 6.

\subsection{Optimizing other functions}

Dijkstra's algorithm finds cost-optimal paths through a directed graph whose edges have arbitrary positive weights. A detailed description can be found for example in [Gould, 1988; Bondy \& Murty, 1976]. The time complexity of Dijkstra's algorithm is $O\left(n^{2}\right)$, where $n$ is the number of vertices

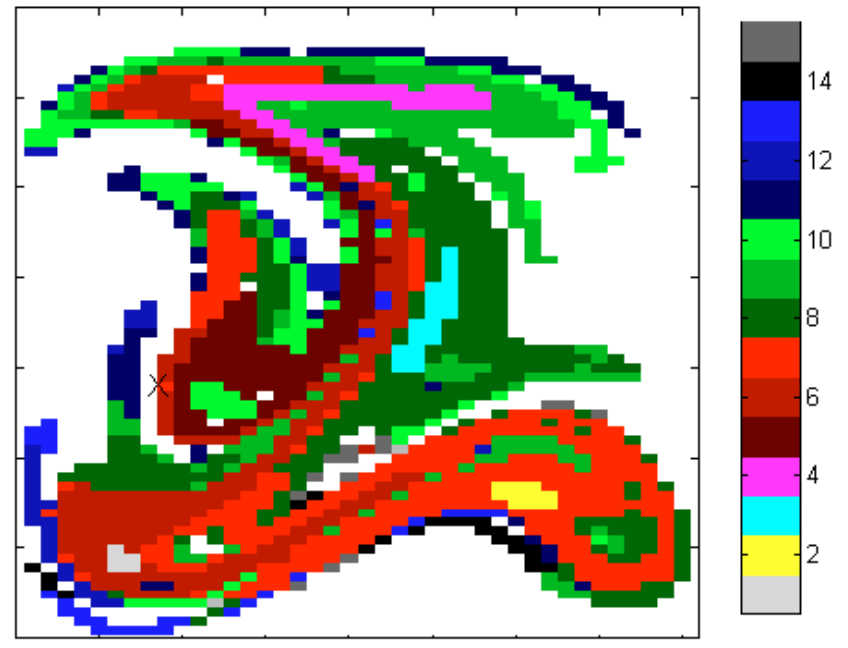

Fig. 3. Targeting the Duffing map using a grid of size $0.1 \times 0.1$. Colors indicate the number of steps through the directed graph required to reach each given colored box, corresponding to an $\varepsilon$-chain of a certain length. These can be forced to a real orbit of the differential equation by parameteric feedback control.

in the graph. This time requirement is small compared to the $O\left(M^{2}\right)$ time needed to construct the graph from a test orbit of length $M$-iterates.

A natural choice for a positive cost function $F(x)$ is the time of flight from $x$ to its next return to the surface of section for a Poincaré return map. Dijkstra's algorithm can be used to find an orbit on the Poincaré section that corresponds to a very fast orbit in the original flow. In our example, the $2 \pi$-stroboscopic mapping method makes for a special case in which time of flight is uniform. In other common dynamical systems, such as Rossler, Lorenz, Restricted-Three-Body-Problem, etc., time of flight to Poincare' surface is not expected to be uniform, and therefore iterate optimization is distinct from time optimization.

Other cost functions might be natural, such as money, fuel, etc. A cost function can also be chosen to design a control strategy to avoid prespecified regions of the chaotic attractor. To avoid a "bad region," a directed graph constructed from a test orbit on the full attractor, vertices corresponding to undesirable regions on the attractor can be eliminated by assigning an infinite weight to the incoming edges. This is equivalent to assigning zero probability of transition, which is how we described the construction of the Cantor-like nonattracting chaotic saddles described in [Bollt et al., 1997]. 
Dijstra algorithm allows optimal paths to be designed for any problem whose coarse-grained transitions can be stated with positive weights.

\section{Choosing Invariant Measure: IFPP}

\subsection{Designing a desirable $F-P$ operator}

In [Bollt, 2000a], we first stated the following control problem, called the Inverse Frobenius-Perron Problem. So that this can be a self-contained presentation, we repeat some of that pertinant information here, along with some new information.

Consider $f: M \rightarrow M, M \subset \Re^{n}$, to be a smooth nonsingular dynamical system, which for our purposes may be derived by Poincare' surface of section from say, Eq. (1). Suppose we wish to control a desirable probability density function (pdf) $\rho^{*} \in L^{1}(M)$, which is different from the (pdf) of $f$ which in general we do not even need to assume exists. Our goal will be to construct a $C^{0}$ nearby transformation $f+\delta f$ such that this new dynamical system has the desired pdf $\rho^{*}$. We have an inverse problem to modify the Frobenius-Perron operator.

Since our target invariant density $\rho^{*}$ is the fixed point of the Frobenius-Perron eigenequation, $P_{f+\delta f}\left[\rho^{*}\right]=\rho^{*}$, our approach will be to work with an approximation of the Frobenius-Perron operator of $f$. Given $f$, and a uniform partition $\mathcal{G}_{n}$, a probability matrix $S_{n \times n}$ may derived by the Ulamlike Eq. (5), or more efficiently by observing a test orbit and using Eq. (6). Roughly said, the dominant $(\lambda=1)$ eigenvector $v_{n}$ of $S_{n \times n}$ gives the long term statistics of the Markov process described by the directed graph. Likewise, it is expected to approximate the long term probability density (pdf) of $f$, in a weak limit sense. We do not give here the sufficient conditions for any of the several theorems [Li, 1976; Froyland, 1995], Froyland2, Ding-Zhou, [Góra \& Boyarsky, 1997] which have successfully proven for which transformations the last sentence is true, because we are not really concerned with invariant density of $f$, but rather that of our control target $f+\delta f$. We have given [Bollt, 2000a] mild sufficient conditions under which the new transformation $f+\delta f$ has the new probability matrix $S_{n \times n}^{*}$ as its Frobenius-Perron operator. One such sufficient condition is that $S_{n \times n}^{*}$ is irreducible (the directed graph allows walks between any two vertices) and aperiodic, which allows us to produce a piecewise affine transformation $f^{*}$ such that $P_{f^{*}} \equiv S_{n \times n}^{*}$.
Stated in terms of matrices, our control problem is as follows. Given a stochastic matrix $S_{n \times n}$, produce a new probability matrix $S_{n \times n}^{*}$ such that a desired probability vector $u_{n}$ is stationary, $u_{n}$. $S_{n \times n}^{*}=u_{n}$, where $u_{n}$ approximates $\rho^{*}$ on the grid. To guarantee $C^{0}$ small variations between the new transformation $f^{*}$ and the old transformation $f$, we require that $S_{n \times n}^{*}$ respect the grammar of $S_{n \times n}$, meaning $\left[S_{n \times n}\right]_{i, j}=0 \Rightarrow\left[S_{n \times n}^{*}\right]_{i, j}=0$. In general this is not a well posed problem. As such, this allows us a certain amount of freedom. The statements of, (i) invariance $\left(S_{n \times n}^{*} \cdot u_{n}=u_{n}\right)$, (ii) grammar respect, and (iii) that $S_{n \times n}^{*}$ be a probability matrix, each can be described by linear constraint of order no larger than $n \times n^{2}$.

Our constraints [Bollt, 2000a] may be stated in the following abreviated notation inspired from a recent paper by Gora and Boyarsky [1999]. First let $\mathbf{s}_{n^{2}}^{*}$ be an $n^{2} \times 1$ vector whose entries come from $n \times n$ unknown matrix $S_{n \times n}^{*} ; S_{i, j}^{*} \mapsto s_{[(j-1) n+i+1]}^{*}$, for $1 \leq i, j \leq n$, which reads each row of $S_{n \times n}^{*}$, in turn, into the vector $\mathbf{s}_{n^{2}}^{*}$.

1. Target Invariance. Rewrite the invariance constraint $u_{n} \cdot S_{n \times n}^{*}=u_{n}$ to emphasize that in general the target density vector $u_{n}$ is known and the matrix $S_{n \times n}^{*}$ is unknown by the equivalent system $K^{(1)} \cdot \mathbf{s}_{n^{2}}^{*}=B^{(1)}$ in which the matrix is known, and the vector is the unknown. Map each of the $n$ constraint values of the $n \times 1$ vector $u_{n}$ into an $n \times n^{2}$ "place-holder" matrix $K^{(1)}$; let $K_{i, j}^{(1)}=\left[u_{n}\right]_{j}$, for $(i-1) n+1 \leq j \leq(i)(n)$, $i=1,2, \ldots, n$, and otherwise $K_{i, j}^{(1)}=0$. Let $B^{(1)}=u_{n}$.

2. Grammar respect. $\left[S_{n \times n}\right]_{i, j}=0 \Rightarrow\left[S_{n \times n}^{*}\right]_{i, j}=0$, can be stated as a linear constraint by marking each of the $k, 1 \leq k \leq n^{2}$, zero entries of $S_{n \times n}$ into a "place-holder" matrix $K^{(2)}$. Let $K^{(2)}$ be a $k \times n^{2}$ matrix defined such that each $m$ th row of $K^{(2)}$ has a 1 for each zero entry of $S_{n \times n}$; let $1 \leq m \leq k$ index the $m$ th zero entry of $\left[S_{n \times n}\right]_{i, j}>0$, and define $K_{[m, i+n(j-1)]}^{(2)}=1$, and let all other entries of $K^{(2)}$ be zero. Let $B^{(2)}=\mathbf{0}$ be the $k \times 1$ vector. Then grammar invariance is now the linear constraint on $\mathbf{s}_{n^{2}}^{*}$ : $K^{(2)} \cdot \mathbf{s}_{n^{2}}^{*}=B^{(2)}$.

3. Stochastic. If $S_{n \times n}^{*}$ is constrained to be a probability matrix, it must row sum to 1 . Define $K^{(3)}$ to be $n \times n^{2}$ such that the $i$ th row of $K^{(3)}$ corresponds to the $i$ th row sum of $S_{n \times n}^{*}$, recorded in the vector of matrix entries $\mathbf{s}_{n^{2}}^{*}$. Let 
$K_{i, j}^{(3)}=1$ if for each $1 \leq i \leq n$, and for each $1+(i-1) n \leq j \leq 1+(i)(n)$, and all other entries of $K^{(3)}$ are zero. To force each row sum to be 1 , choose $B^{(3)}=\mathbf{1}$, the $n \times 1$ vector of ones. We have now the linear constraint $K^{(3)} \cdot \mathbf{s}_{n^{2}}^{*}=B^{(3)}$.

The intersections of these constraints can now be stated as a single linear constraint,

$$
K \cdot \mathbf{s}_{n^{2}}^{*}=B,
$$

where we simply "stack" the above constraints 1-3; let $K=\left[K^{(1)}: K^{(2)}: K^{(3)}\right]^{T}$ and $B=\left[B^{(1)}: B^{(2)}\right.$ : $\left.B^{(3)}\right]^{T}$. See the worked example in Sec. 5.1.1.

Generally, there is freedom in solving for $\mathbf{s}_{n^{2}}^{*}$ since $K$ is usually far from full rank. In our previous work [Bollt, 2000a], we took the point of view that a particular solution can be found by minimizing $\left\|S_{n \times n}-S_{n \times n}^{*}\right\|$ by the Penrose-Pseudo Inverse (least squares) solution in the $l^{2}$ norm or in [Bollt, $2000 \mathrm{~b}]$ by repeated linear programming in the $l^{\infty}$ norm. Equation (8) will always have a minimal solution, but if $S_{n \times n}^{*}$ is to be a real probability matrix, it is necessary to require entry-wise of the matrix that,

$$
\mathbf{0} \leq \mathbf{s}_{n^{2}}^{*} \leq \mathbf{1} .
$$

In our formulation of the problem [Bollt, 2000a, 2000b], constraint Eq. (9) can only be used as an $a$ posteriori check of success; either our exact target density is attainable within the fine grid partition, or it is not. We gave theorems which indicated that one expects that a coarser-grid is expected to admit a solution to Eqs. (8) and (9). In [Bollt, 2000a], we interpreted Eq. (8) as defining a hyperplane in $\Re^{n^{2}}$, since $K$ is generally not of full rank. Continuing with this geometric point of view, Eq. (9) defines a unit hyperbox, and hence we stated that a useful solution exists iff hyperplane Eq. (8) pierces the unit box Eq. (9).

In our previous work [Bollt, 2000a, 2000b], we discussed minimization of the variation to the matrix approximation of the $\mathrm{F}-\mathrm{P}$ operator,

$$
\begin{aligned}
C\left(\mathbf{s}_{n^{2}}^{*}\right) & =\left\|S_{n \times n}-S_{n \times n}^{*}\right\| \\
& =\sqrt{\sum_{1 \leq i, j \leq n}\left(S_{i, j}-S_{i, j}^{*}\right)^{2}},
\end{aligned}
$$

which is a least squares problem, best solved by Singular Value Decomposition. We discussed that this required choosing a given target density. We presented controllability theorems that if no good minimum of an arbitrary target density exists within constraints, one must manually modify the target, $\mathbf{s}_{n^{2}}^{*}$. Gora and Boyarsky have recently made an important modification [Góra \& Boyarsky, 1999] to our control problem by using another cost function,

$$
\begin{aligned}
C\left(\mathbf{s}_{n^{2}}^{*}\right) & =\left\|\mathbf{u}_{n}-\mathbf{v}_{n}\right\|_{2} \\
& =\sqrt{\sum_{1 \leq i \leq n}\left(u_{i}-v_{i}\right)^{2}},
\end{aligned}
$$

the Euclidean distance between the target density $\mathbf{v}_{n}$ and the dominant vector $\mathbf{u}_{n}$ of $S_{n \times n}^{*}$. We can minimize Eq. (11) subject to both types of linear constraints Eqs. (8) and (9), by applying standard minimization packages. For example, the routine constr which is built into MatLab's Optimization Package [Colemann et al., 1999]; see also [Press et al., 1986]. Using the cost function Eq. (11), Gora and Boyarsky minimize error between target density $\mathbf{v}$, and the attainable density $\mathbf{u}$. They remarked that such an approach to optimization is favorable to our less flexible Pseudo-Inverse solution which insists on the invariance property in Eq. (8) but sometimes gives the unsatisfactory answer that no solution exists which also satisfies Eq. (9). The more general optimization approach finds the probability matrix with the dominant eigenvector $v_{n}$ as close as possible (if not exact) to the target dominant vector $\mathbf{s}_{n^{2}}^{*}$, within matrix constraints.

For a more detailed description of setting-up Eq. (8), please see [Bollt, 2000a, 2000b] and [Góra \& Boyarsky, 1999]. We give a worked example in the following subsection.

\subsubsection{Example of designing a desirable $F-P$ operator}

The linear algebra formalism of the last section is perhaps confusing without a concrete example, which we provide in this subsection. Exposing a small example matrix is facilitated both by choosing a low dimensionional example and of extreme coarseness. Albiet, a finer grid is likely more useful in practice. Hence, we choose the one-dimensional successive maxima map derived from the Lorenz differential equations [Lorenz, 1963], rather than our primary Duffing oscillator example.

We choose an $n=8$ element grid, over the range of the $z$-variable of the Lorenz equations. See the caption Fig. 4. This one-dimensional map gives 
an approximation,

$$
S_{8 \times 8}=\left(\begin{array}{cccccccc}
0.6957 & 0.3043 & 0 & 0 & 0 & 0 & 0 & 0 \\
0 & 0.5455 & 0.4545 & 0 & 0 & 0 & 0 & 0 \\
0 & 0 & 0.4202 & 0.5798 & 0 & 0 & 0 & 0 \\
0 & 0 & 0 & 0.1057 & 0.4978 & 0.3700 & 0.0264 & 0 \\
0 & 0 & 0 & 0 & 0.2566 & 0.3009 & 0.3628 & 0.0796 \\
0 & 0 & 0.0197 & 0.6184 & 0.3618 & 0 & 0 & 0 \\
0 & 0.1818 & 0.8182 & 0 & 0 & 0 & 0 & 0 \\
0.3333 & 0.6667 & 0 & 0 & 0 & 0 & 0 & 0
\end{array}\right),
$$

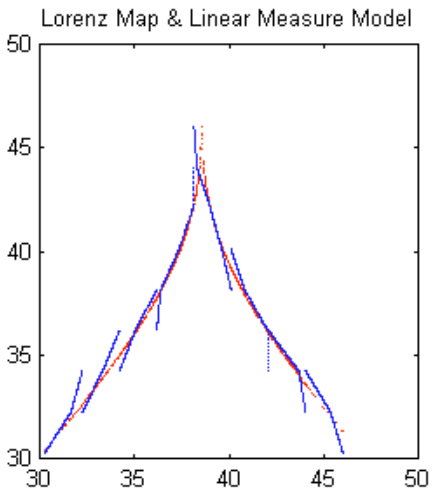

(a)

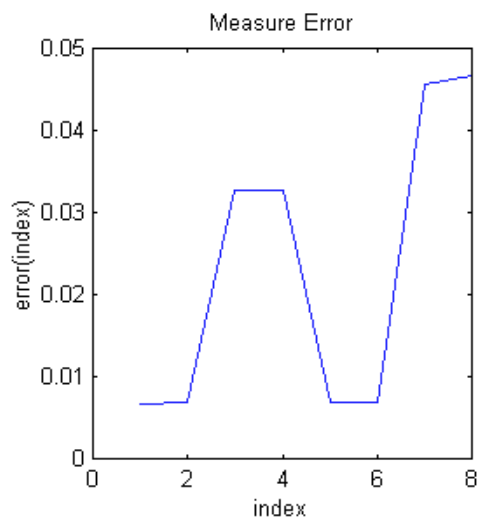

(d)

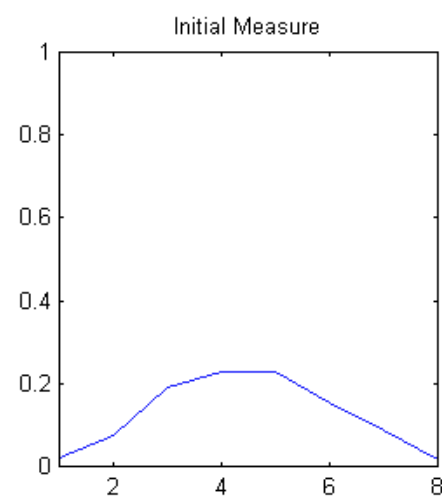

(b)

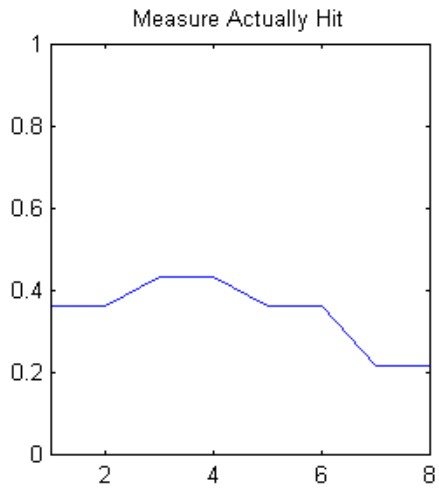

(e)

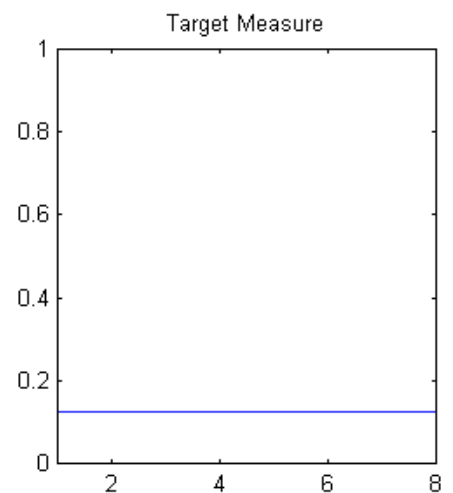

(c)

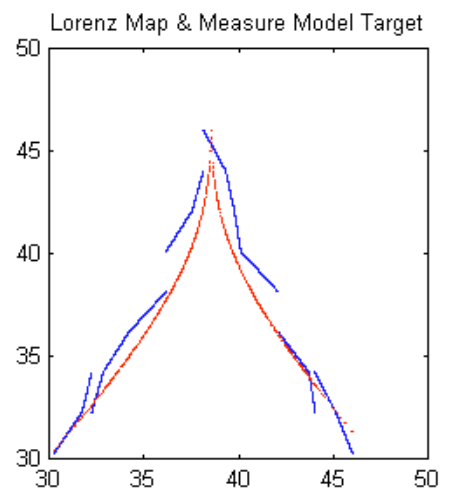

(f)

Fig. 4. IFPP and the Lorenz successive maxima map. The well-known [Lorenz, 1963] Lorenz equations, $\dot{x}=10(y-x)$, $\dot{y}=x(28-z)-y, \dot{z}=x y-(8 / 3) z$, create the 1-D map shown in red in (a) when successive local maxima of $z(t)$ are plotted $z\left(t_{\text {Next Max }}\right)=f\left(z\left(t_{\text {Local Max }}\right)\right.$. Using an $n=8$ element grid, the approximated uncontrolled invariant density is shown in (b). We choose the uniform invariant density as our target shown in (c). The closest possible minimization of Eq. (11) subject to Eqs. (8) and (9) does not exactly hit the uniform target density shown in (c), since uniform is not an invariant density of the original transformation. In (d) we show the error between uniform (c), and the density actually found by optimal matrix $S_{8 \times 8}^{*}$, and in (e) we plot this density. In (a) and (f) we show the original Lorenz map in red, and its "measure-models" in blue. That is, the blue piecewise linear maps shown are found by the inverse Ulam problem, and are those linear transformations which have exactly a specific matrix, $S_{8 \times 8}$ and $S_{8 \times 8}^{*}$ respectively as their Frobenius-Perron operators on the chosen uniform $n=8$ element grids. Hence the blue curve shown in (f) is our measure target. 
to the Frobenius-Perron operator on the successive maxima surface of section, and using the $n=8$ element grid. Its dominant eigenvector is shown in Fig. 4(b). We wish to target a uniform density shown in Fig. 4(c). Now we can discuss points 1-3 of formulating IFPP as a linearly constrained optimization problem.

to be,

Choose the target eigenvector of $\mathbf{u}_{8} \cdot S_{8 \times 8}=\mathbf{u}_{8}$

$$
\mathbf{u}_{8}=\left(\begin{array}{llllllll}
\frac{1}{8} & \frac{1}{8} & \frac{1}{8} & \frac{1}{8} & \frac{1}{8} & \frac{1}{8} & \frac{1}{8} & \frac{1}{8}
\end{array}\right)^{t} .
$$

Let us define the unknown vector of the entries of $S_{8 \times 8}^{*}$ to be the vector unknowns $\mathbf{s}_{64}^{*}$, where the first row of the matrix $S_{8 \times 8}^{*}$ is the first 8 entries of $\mathbf{s}_{64}^{*}$. The second row of $S_{8 \times 8}^{*}$ is then the next 8 entries of $\mathbf{s}_{64}^{*}$, that is entries $9-16$, etc.

Now we are in a position to explicitly describe each of the three parts from the previous section, in building the rules of our linear constraint Eq. (8).

1. Target Invariance. $\mathbf{u}_{8} \cdot S_{8 \times 8}^{*}=\mathbf{u}_{8}$ can be written $K_{8 \times 64}^{(1)} \cdot \mathbf{s}_{64}^{*}=B_{8}^{(1)}$, to emphasize that the entries of $S_{8 \times 8}^{*}$ are unknown, by letting

$$
K_{8 \times 64}^{(1)}=\left(\begin{array}{llllllll}
\mathbf{u}_{8}^{t} & \mathbf{0}_{8}^{t} & \mathbf{0}_{8}^{t} & \mathbf{0}_{8}^{t} & \mathbf{0}_{8}^{t} & \mathbf{0}_{8}^{t} & \mathbf{0}_{8}^{t} & \mathbf{0}_{8}^{t} \\
\mathbf{0}_{8}^{t} & \mathbf{u}_{8}^{t} & \mathbf{0}_{8}^{t} & \mathbf{0}_{8}^{t} & \mathbf{0}_{8}^{t} & \mathbf{0}_{8}^{t} & \mathbf{0}_{8}^{t} & \mathbf{0}_{8}^{t} \\
\mathbf{0}_{8}^{t} & \mathbf{0}_{8}^{t} & \mathbf{u}_{8}^{t} & \mathbf{0}_{8}^{t} & \mathbf{0}_{8}^{t} & \mathbf{0}_{8}^{t} & \mathbf{0}_{8}^{t} & \mathbf{0}_{8}^{t} \\
\mathbf{0}_{8}^{t} & \mathbf{0}_{8}^{t} & \mathbf{0}_{8}^{t} & \mathbf{u}_{8}^{t} & \mathbf{0}_{8}^{t} & \mathbf{0}_{8}^{t} & \mathbf{0}_{8}^{t} & \mathbf{0}_{8}^{t} \\
\mathbf{0}_{8}^{t} & \mathbf{0}_{8}^{t} & \mathbf{0}_{8}^{t} & \mathbf{0}_{8}^{t} & \mathbf{u}_{8}^{t} & \mathbf{0}_{8}^{t} & \mathbf{0}_{8}^{t} & \mathbf{0}_{8}^{t} \\
\mathbf{0}_{8}^{t} & \mathbf{0}_{8}^{t} & \mathbf{0}_{8}^{t} & \mathbf{0}_{8}^{t} & \mathbf{0}_{8}^{t} & \mathbf{u}_{8}^{t} & \mathbf{0}_{8}^{t} & \mathbf{0}_{8}^{t} \\
\mathbf{0}_{8}^{t} & \mathbf{0}_{8}^{t} & \mathbf{0}_{8}^{t} & \mathbf{0}_{8}^{t} & \mathbf{0}_{8}^{t} & \mathbf{0}_{8}^{t} & \mathbf{u}_{8}^{t} & \mathbf{0}_{8}^{t} \\
\mathbf{0}_{8}^{t} & \mathbf{0}_{8}^{t} & \mathbf{0}_{8}^{t} & \mathbf{0}_{8}^{t} & \mathbf{0}_{8}^{t} & \mathbf{0}_{8}^{t} & \mathbf{0}_{8}^{t} & \mathbf{u}_{8}^{t}
\end{array}\right)
$$

In general, Eq. (14) may be written more compactly using the Kronecker "tensor product" $\otimes$, [Fallet, 1996], which has become popular lately in the syncronization literature under the name "direct product," [Pecora \& Carroll, 1998; Pecora et al., 1997] (in formulating the "masterstability function"). Let,

$$
K_{n \times n^{2}}^{(1)}=\mathbf{I}_{n \times n} \otimes \mathbf{u}_{n}^{t} .
$$

We use the notation that $\mathbf{0}_{n}$ is the $n \times 1$ vector of zeros, and $\mathbf{I}_{n \times n}$ is the identity matrix. Finally, let $B_{8}^{(1)}=\mathbf{u}_{8}$ be the target vector.
2. Grammar respect is also easily written into a linear constraint $K_{k \times n^{2}}^{(2)} \cdot \mathbf{s}_{64}^{*}=B_{k}^{(2)}$, as described in the previous section. Specifically, we need each entry of $S_{8 \times 8}^{*}$ to be zero whenever the corresponding entry of $S_{8 \times 8}$ is also zero, and correspondingly in $\mathbf{s}_{64}^{*}$. For example, observe that the $(i, j)=(1,3)$ entry of $S_{8 \times 8}$, in Eq. (12), is zero. Calling this the first zero, we write into the first row of the place-holder matrix $K_{k \times n^{2}}^{(2)}$ by letting the first row of $K_{k \times n^{2}}^{(2)}$ be all zeros, except in the third column. Showing only this first row,

$$
K_{43 \times 64}^{(2)}=\left(\begin{array}{cccccc}
0 & 0 & 1 & 0 & \rightarrow & 0 \\
* * & \rightarrow & & & &
\end{array}\right)
$$

Counting the zero entries of $S_{8 \times 8}$ in Eq. (12) yields $k=43$, and hence we choose $B_{43}^{(2)}=\mathbf{0}_{43}$ to be the $43 \times 1$ zero matrix. It is easy to see that the matrix multiplication in the equation $K_{43 \times 64}^{(2)} \cdot \mathbf{s}_{64}^{*}=B_{43}^{(2)}$ will force the third entry of $\mathbf{s}_{64}^{*}$ corresponding to the $(i, j)=(1,3)$ entry of $S_{8 \times 8}$ to be zero, as required. The other zero's of $S_{8 \times 8}$ may be similiarly written into $K_{43 \times 64}^{(2)}$ as described in the previous section.

3. Stochastic. As already stated in the previous section, constraining $S_{8 \times 8}^{*}$ to be a stochastic matrix is partially satisfied by requiring that its column sums to one. The reshaping of these unknown matrix entries to the vector $\mathbf{s}_{64}^{*}$ requires a linear constraint in which the $j$ th row of $K$ selects each element of the $j$ th column of $S_{8 \times 8}^{*}$ from its reshaping as $\mathbf{s}_{64}^{*}$. For our example, we let, $w_{1}=\left(\begin{array}{llllllll}1 & 0 & 0 & 0 & 0 & 0 & 0 & 0\end{array}\right)$, $w_{2}=\left(\begin{array}{llllllll}0 & 1 & 0 & 0 & 0 & 0 & 0 & 0\end{array}\right)$, and $w_{3}=$ $\left(\begin{array}{llllllll}0 & 0 & 1 & 0 & 0 & 0 & 0 & 0\end{array}\right)$, etc., be $1 \times 8$ matrices. Showing the first few rows only,

$$
K_{8 \times 64}^{(3)}=\left(\begin{array}{llllllll}
w_{1} & w_{1} & w_{1} & w_{1} & w_{1} & w_{1} & w_{1} & w_{1} \\
w_{2} & w_{2} & w_{2} & w_{2} & w_{2} & w_{2} & w_{2} & w_{2} \\
w_{3} & w_{3} & w_{3} & w_{3} & w_{3} & w_{3} & w_{3} & w_{3} \\
* * & \rightarrow & & & & & &
\end{array}\right)
$$

Again the tensor product permits a compact notation, as follows,

$$
K_{n \times n^{2}}^{(3)}=\mathbf{1}_{n}^{t} \otimes \mathbf{I}_{n \times n} .
$$

Choosing $B_{n}^{(3)}=\mathbf{1}_{n}$ to be the $n \times 1$ matrix of all ones, it is easy to see that the linear constraint 
$K_{n \times n^{2}}^{(3)} \cdot \mathbf{s}_{n}^{*}=B_{n}^{(3)}$ forces corresponding column sums of corresponding entries of $S_{8 \times 8}^{*}$ to be one. In this example, $n=8$.

Note that Eqs. (15) and (18) are new and simplified representations of Eqs. (14) and (17) respectively.
Now stacking each of these three linear constraints yields the intersection equality constraint, Eq. (8). Finally, solving our linear mixed equality and inequality Eqs. (8) and (9) constrained optimization of Eq. (11) does not yield exactly the matrix whose left eigenvector is the uniform target vector Eq. (13). Instead, the optimal solution is the matrix,

$$
S_{8 \times 8}^{*}=\left(\begin{array}{cccccccc}
0.7365 & 0.2635 & 0 & 0 & 0 & 0 & 0 & 0 \\
0 & 0.3060 & 0.6940 & 0 & 0 & 0 & 0 & 0 \\
0 & 0 & 0 & 1.0000 & 0 & 0 & 0 & 0 \\
0 & 0 & 0 & 0 & 0 & 0.6926 & 0.3074 & 0 \\
0 & 0 & 0 & 0 & 0 & 0.1717 & 0.2342 & 0.5941 \\
0 & 0 & 0 & 0 & 1.0000 & 0 & 0 & 0 \\
0 & 0.1657 & 0.8343 & 0 & 0 & 0 & 0 & 0 \\
0.4428 & 0.5572 & 0 & 0 & 0 & 0 & 0 & 0
\end{array}\right)
$$

whose left eigenvector is the best possible within the grammar (and grid). We show the error between target and actually hit invariant densities in Fig. 4(d), and we show the actually hit invariant density shown in Fig. 4(e), which is,

$$
\mathbf{v}^{t}=\left(\begin{array}{llll}
0.1316 & 0.1318 & 0.1577 & 0.1577 \\
0.1318 & 0.1318 & 0.0793 & 0.0783
\end{array}\right)^{t} .
$$

In Figs. 4(a) and 4(f), we show in blue the piecewise linear measure models which we will discuss in more detail in the next section.

\subsection{Designing the dynamical system}

Once the probability matrix $S_{n \times n}^{*}$ has been found, we must formulate a discrete dynamical system $f^{*}$ such that $S_{n \times n}^{*}$ is its Frobenius-Perron operator on the given grid partition $\mathcal{G}_{n}$. The point transformation $f^{*}$ serves as our local model, which we consider here to be our local targets to be achieved by feedback control. These are small by construction. This point is made explicitly obvious in the low dimensional Lorenz map example depicted in Fig. 4. We first briefly recall [Bollt, 2000a] the deterministic solution, which we call IUP, and then we formulate the stochastic solution which is both simpler, and more stable in the present context of feedback control.

\subsubsection{Inverse Ulam problem}

Given a probability matrix $S_{n \times n}^{*}$ and a partition $\mathcal{G}_{n}$, it is straightforward to construct a piecewise affine transformation $f^{*}$ which is consistent with the Frobenius-Perron operator Eq. (3). In 1-D, on each grid element, it is only required that the transformation's derivatives come from one over the matrix entry which can easily be done so that $f^{*}$ is Markov; see Fig. 4 for pictoral insight, and [Góra \& Boyarsky, 1997; Bollt, 2000a] for details. In multi-dimensions, the appropriate piecewise affine transformation can also be easily constructed by mapping horizontal strips in $G_{i}$ linearly onto vertical strips in $G_{j}$, (which guarantees the Markov property [Bowen, 1975]), in such a way that change in volume of the strips, is given by the determinant of the Jacobian derivative. This agrees with Eq. (3) and the target matrix entry $\left[S_{n \times n}^{*}\right]_{i, j}$. Hence correspondance between the matrix $S_{n \times n}^{*}$, and the Frobenius-Perron operator $P_{f^{*}}$ of the constructed transformation is ensured.

We have reviewed here only in the briefest of details the deterministic solution of the IFPP by IUP, since it is our opinion that such is not the best local model for feedback control of a differential equation. This is because, considering that probabilities of transitions depends so sensitively on position, from strip to strip, any slight error in targeting $f^{*}(x)$ by feedback control will affect the next transition, and so on. It is feasible that a consistent error in targeting $f^{*}(x)$ could bias the density results unexpectedly. The IFS stochastic model of $f^{*}$ below is more immune to such error, since as long as the controlled next iterate is anywhere within the 
target box, then statistics are exactly as expected and desired. Note that the piecewise linear maps shown in Fig. 4 are only offered as measure models, as we use an IFS for actual feedback control.

\subsubsection{Iterated Functions System (IFS)}

Given a probability matrix $S_{n \times n}^{*}$ and the partition $\mathcal{G}_{n}$, an iterated functions system model [Barnsley, $1988]$ for $f^{*}$ is realized as follows. For each $x$, determine $i$ such that $x \in G_{i}$. Choose a random number $z$ uniformly in $[0,1]$ and determine $j$ cumulatively, by requiring that

$$
\sum_{k=1}^{j-1}\left[S_{n \times n}^{*}\right]_{i, k}<z \leq \sum_{k=1}^{j}\left[S_{n \times n}^{*}\right]_{i, k},
$$

or $j=1$ if $z \leq\left[S_{n \times n}^{*}\right]_{i, 1}$. Next, simply assign the center of box $G_{j}$ as the next iterate target; let $f^{*}(x)=\overline{G_{j}}$. Note that choosing the center of $G_{j}$ best allows immunity to small errors.

Note that this stochastic model of $f^{*}$ is neither completely deterministic, nor completely random. Such is typical of an IFS. We have essentially defined a stochastic process which is highly constrained to follow the large-scaled transitions of a given directed graph. The process is deterministic on the global scale, and random on the small scale, which of course sensitively affects the long term global scale process.

In the general and realistic settings of a slightly and additively randomly perturbed dynamical system $x_{n+1}=f(x)+\mathbf{g}$ where $\mathbf{g}$ is some random variable with small standard deviation, such a coarse-grained IFS model is not only natural, but perhaps most realistic since small scaled specification of transitions are blurred by the noise $\mathbf{g}$.

\section{Multi-Parametric Control Closes an $\varepsilon$-Chain in a Flow}

Given a discrete transformation $\mathbf{f}$, existence of a new transformation $\mathbf{f}^{*}$ with desirable invariant density may be considered to be an open-loop control strategy. Such is not feasible for a differential equation. Rather, $\mathbf{f}$ and $\mathbf{f}^{*}$ exist simply as a snap-shot of the flow and flow targets on Poincare' surface of section. For a flow, $\mathbf{f}^{*}$ should be considered to be the function of all possible next iterate targets, over $M$, which will achieve some desirable long-term steady state, described as an invariant density $\rho^{*}$ of $\mathbf{f}^{*}$. To achieve $\mathbf{f}^{*}(\mathbf{x})$ in a differential equation, pointwise for each given phase-state $\mathbf{x}$, we choose to use constant parametric feedback as our control

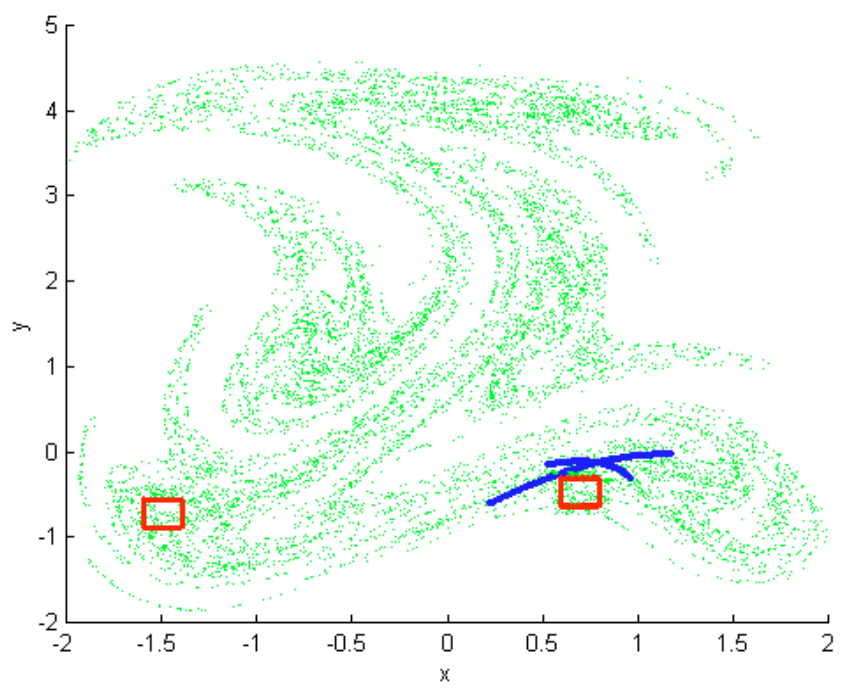

(a)

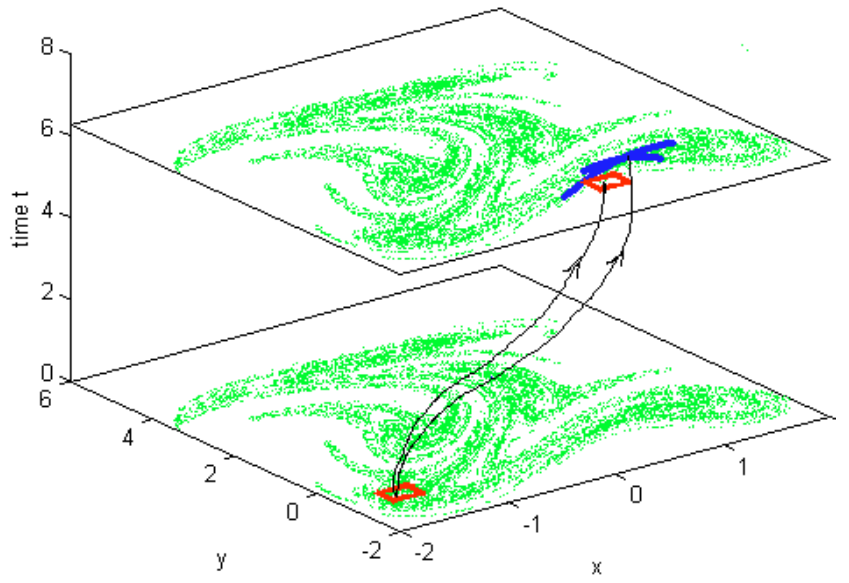

(b)

Fig. 5. Observed next responses due to paramater variations of the Duffing oscillator: $x^{\prime \prime}+a x^{\prime}+x^{3}-x=b \sin (t)$, where $\lambda_{0}=\left(a_{0}, b_{0}\right)=(0.02,3)$. (a) Dots show $N=10000$ iterates of $2 \pi$-stroboscopic map. Bold squares show "from" and "to" vertices, in this (overly-large characature) grid. Blue bold crossed curves show observed next responses due to maximal variations $|\delta \lambda|=(|\delta a|,|\delta b|) \leq(0.02,0.25)$, where either $\delta a$ or $\delta b$ is varied separately, while the other is held fixed. With these blue curves, we see a spanning range of possible next targets due to a maximal parameter variation $\mid \delta \lambda$. (b) Characature of flow between piercings of uncontrolled $\mathbf{f}_{\delta \lambda}$, and controlled differential equation $\mathbf{f}_{\delta \lambda+\lambda_{0}}$, to target box. 
plant. Such is straightforward, since by construction $\left|\mathbf{f}^{*}(\mathbf{x})-\mathbf{f}(\mathbf{x})\right|$ is uniformly small.

Parameteric feedback control of next iterate response is realized as follows, on Poincaré surface. Assuming an initial condition $\mathbf{x}$ and our target response is $\mathbf{x}_{\mathrm{want}}=\mathbf{f}^{*}(\mathbf{x})$ we need to solve the equation,

$$
\mathbf{f}_{\delta \lambda+\lambda_{0}}(\mathbf{x})=\mathbf{x}_{\mathrm{want}}
$$

for the unknown control parameter perturbation vector $\delta \lambda$. Next response variation is a function of both initial position and target, $\delta \lambda\left(\mathbf{x}, \mathbf{x}_{\mathrm{want}}\right)$. In other words, we have to solve a two point boundary value problem (BVP). In general, shooting requires a good initial guess, but as we have already pointed out, $\delta \lambda$ is expected to be small. So we make the initial guess $\delta \lambda=\mathbf{0}$ to seed a Newton's method based shooting algorithm to solve Eq. (22). See Fig. 5.

\section{Numerical Examples}

We will now demonstrate numerical examples of controlling Eq. (1) around nominal parameter values $\lambda_{0}=\left(a_{0}, b_{0}\right)=(0.02,3)$.

\subsection{Numerical examples of stabilizing an optimal path}

With the ability to formulate an optimal path as an $\varepsilon$-chain orbit by methods described in Sec. 4, together with the ability to formulate a true orbit of the flow by time varying parameter perturbations, we can now show an example of optimal targeting of the Duffing oscillator.

We show in Fig. 6 a shortest path, calculated by BFS as in Sec. 4.1, between the empty red box and the blue box labeled " 6 ". Using a $40 \times 40$ grid covering corresponds to $\left(h_{x}, h_{y}\right)=(0.0997,0.1606)$. Each step of the path is likewise indexed and outlined by a blue box, except that the next iterate (and the one to worry about during the calculation of the current parameter perturbation) is the red box labeled "1". We show in the second part of Fig. 6 the control of the just mentioned $\varepsilon$-chain orbit, by hitting the centers of each of the labeled boxes in turn. This requires the calculation of parameter variations $\delta \lambda=(\delta a, \delta b)$ about the nominal values $\lambda_{0}=\left(a_{0}, b_{0}\right)=(0.02,3)$. The blue crosses show the possible range of spacial variations achievable by using either a parameter control $a_{0}-\delta a \leq a \leq a_{0}+\delta a$ and $b=b_{0}$, or $b_{0}-\delta b \leq b \leq b_{0}+\delta b$ and $a=a_{0}$. It is interesting to note that the angle between $\delta a$ and $\delta b$ variations is sometimes very small. It is conceivable that such angle could be approximately zero for some dynamical systems, at some points. In such a case, an extra control parameter would be necessary as the ones used were found to be linearly dependant at certain points. We did not find such a problem with this example.

In Fig. 7, we show the time-dependant parameter variations used, during the time of flight
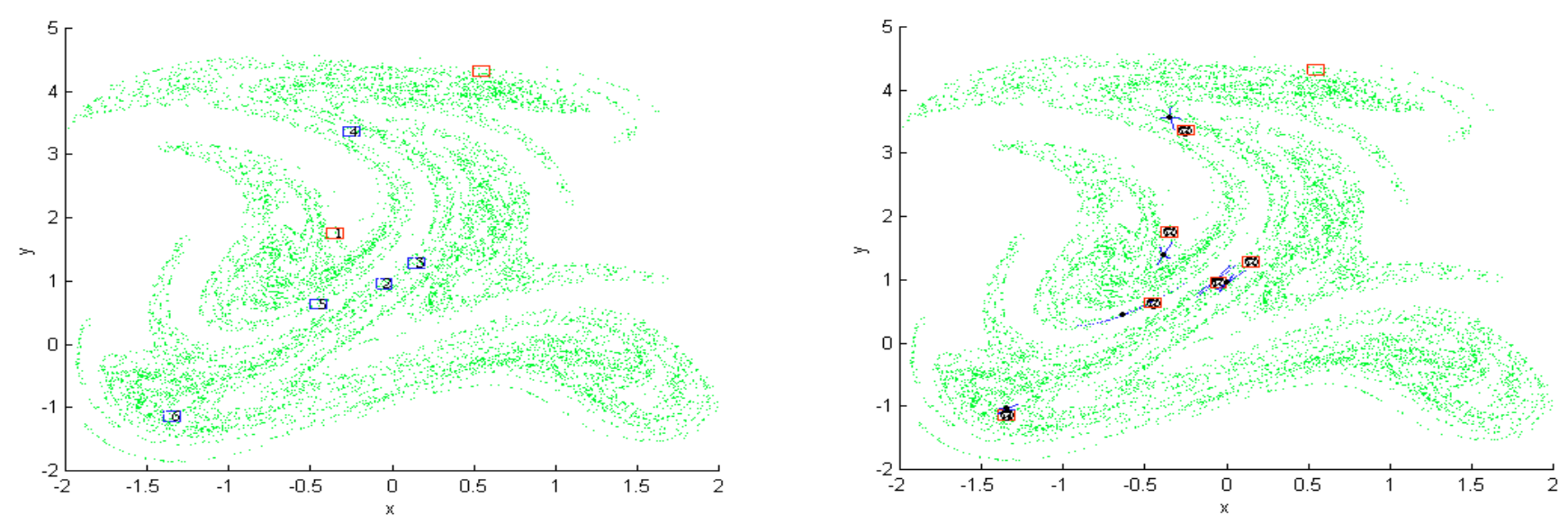

Fig. 6. Targeting the Duffing oscillator, using an $40 \times 40$ grid covering. (Left) Starting in the empty red square, the shortest $\varepsilon$-chain path is found and indexed starting from the empty red square, to the blue square labeled "6". (Right) Using small time-varying parametric perturbations, a true orbit of the flow Eq. (1) hits the centers of each square in turn along the path. The black dot at the center of each blue cross shows where the uncontrolled iteration from the $i$ th box along the path would land instead of hitting the $(i+1)$ th box. The blue crosses through the black dot show the spacial variation range $\delta \mathbf{F}(\mathbf{z})$ possible due to varying each parameter independently. 


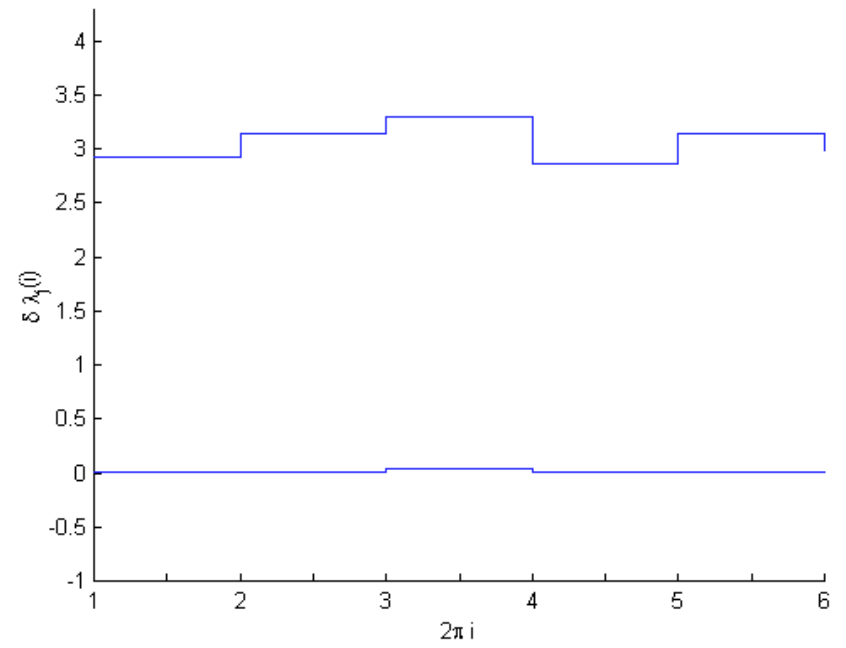

Fig. 7. Time-dependant control parameter variations. The controls used $\delta \lambda=(\delta a, \delta b)$ about the nominal values $\lambda_{0}=$ $\left(a_{0}, b_{0}\right)=(0.02,3)$, to force a true trajectory to follow the 6 -step $\varepsilon$-chain shown in Fig. 6, are small.

between the Poincare surface, to force the true orbit of the ODE, Eq. (1), to follow the $\varepsilon$-chain sequence of boxes depicted in Fig. 6, and calculated by BFS. Continuity of variation of the Poincare map with respect to parameter variation guarantees these perturbations will be small when using a fine grid, and indeed we do observe that our calculated parameter variations are small.

In Fig. 8, we show that using a coarser $10 \times 10$ square grid allows a shorter $\varepsilon$-chain path between the starting square covering the starting point and the final square covering the target. In the limit $\varepsilon \rightarrow 0$, or $n \rightarrow \infty$, we essentially limit to the orbit of the starting point $\mathbf{z}_{a}$. If the target point $\mathbf{z}_{b}$ is already on the orbit of $\mathbf{f}^{N}\left(\mathbf{z}_{a}\right)=\mathbf{z}_{b}$, then "shortest" path is this shortest number of iterates $N$. If the target point $\mathbf{z}_{b}$ is not already on the orbit of $\mathbf{z}_{a}$, then the shortest path length tends to infinity, since we are limiting to allowing no perturbations. On the other end of the scale, if we allow one big grid square covering the attractor, then the minimal path length is zero, since any two points on the attractor, $\mathbf{z}_{a}$ and $\mathbf{z}_{b}$ are already identified, as being in the same square. However, coarser grids require larger parameter perturbations to force. We generally find that the parameter perturbations required to force the controlled flow to follow a coarser $\varepsilon$-chain (smaller value of $n$ ) are larger.

\subsection{Numerical examples of stabilizing invariant densities}

We now show examples of targeting invariant densities of the Duffing oscillator, which have been designed by the IFPP techniques described in Sec. 5 . We control the IFS corresponding to the designed Frobenius-Perron matrix as described in Sec. 5.2.2. Since by construction, the IFS is $\varepsilon$-close (the scale of the grid) to the original dynamical system, small-scaled parametric feedback control forces the flow to follow these coarse-grained trajectories, similarly to the targeting control of the previous subsection.
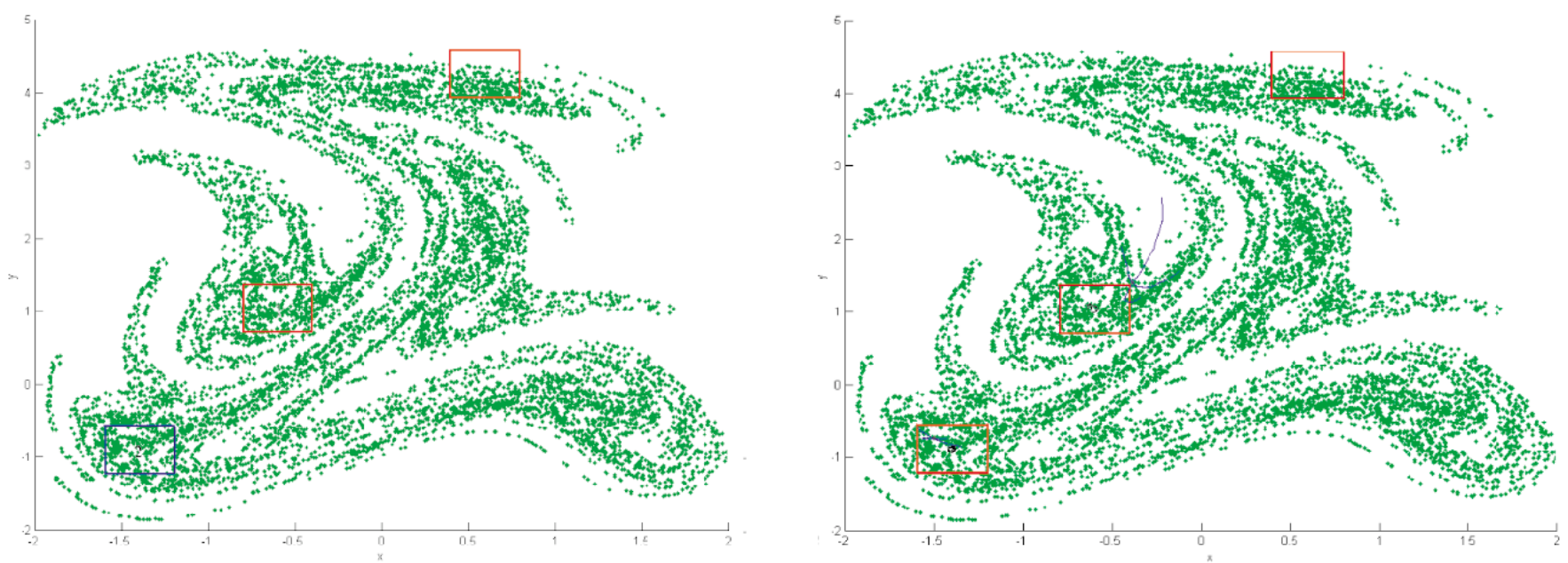

Fig. 8. Targeting the Duffing oscillator, using an $10 \times 10$ square grid covering. Comparison to Fig. 6 shows that the coarser grid allows a shorter path between the starting square covering the starting point and the final square covering the target. Explanation of the graphics is as in Fig. 6. 


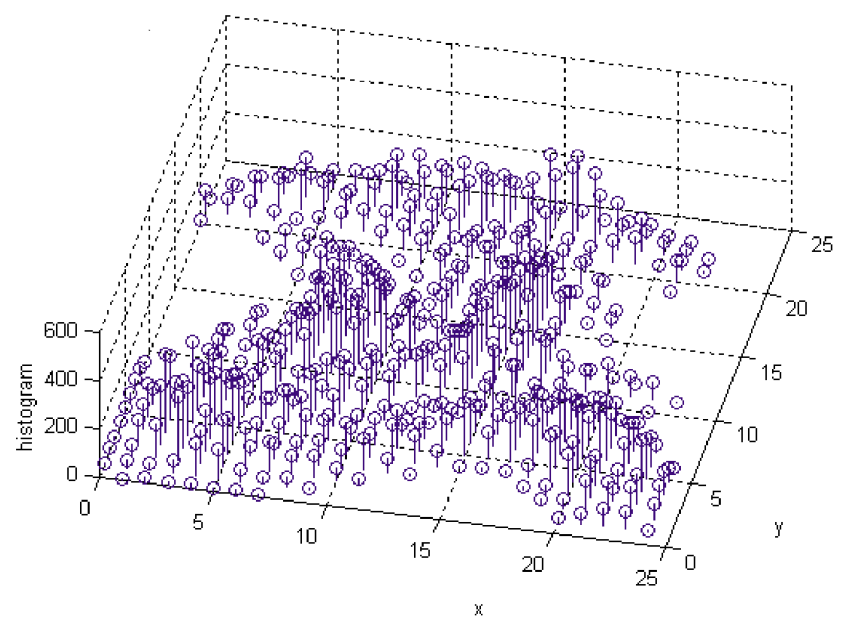

(a)

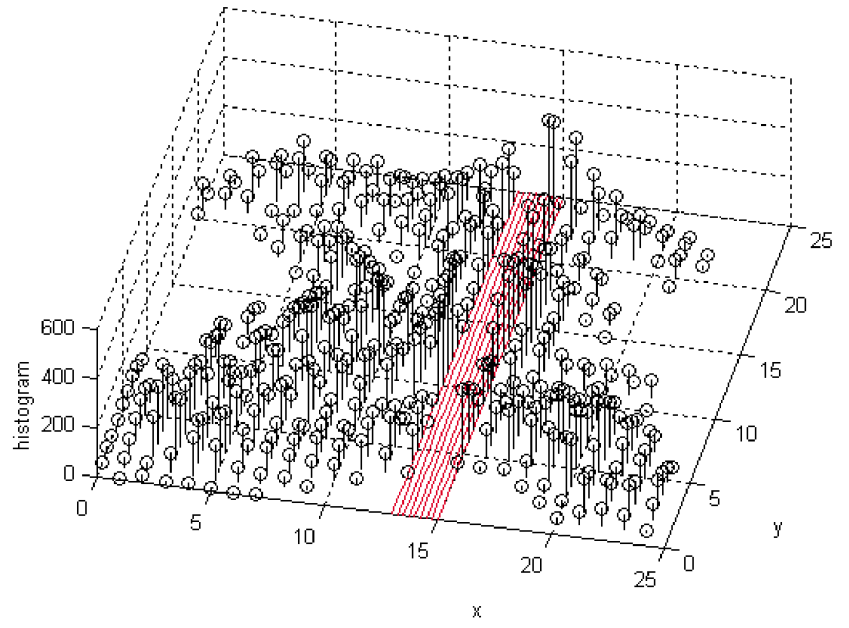

(b)

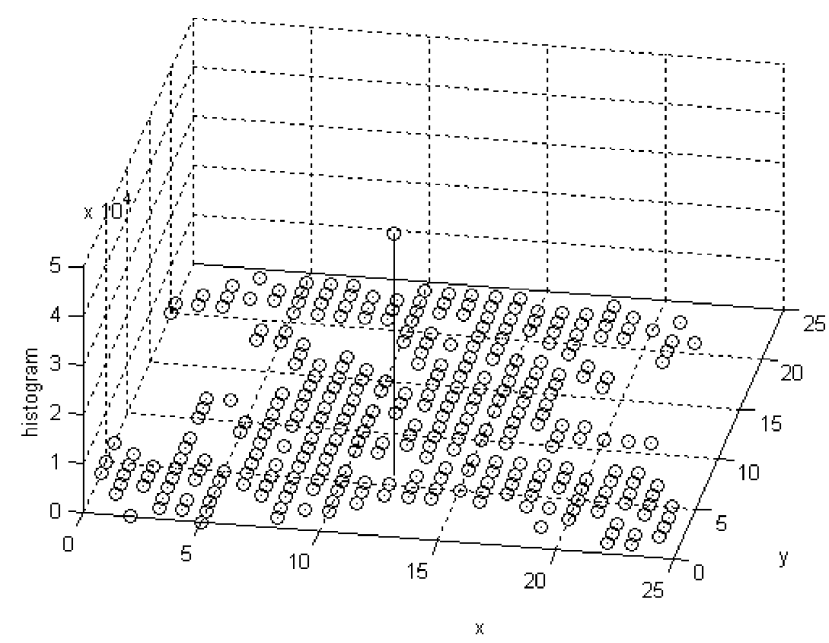

(c)

Fig. 9. (a) Invariant density of the Duffing oscillator, Eq. (1), with parameter values $\lambda_{0}=\left(a_{0}, b_{0}\right)=(0.02,3)$. We have coarse-grained with a $25 \times 25$ grid covering the attractor. (b) Anti-control is realized by choosing an invariant density which avoids the "red-strip" region. (c) Uniformly nearby the Duffing map is a map whose invariant density is supported only over the period-1 of the Duffing map. Stabilizing this nearby map makes the corresponding periodic cycle of the Duffing oscillator globally attracting.

The coarse-grained initial invariant density of the Duffing $2 \pi$-map of the oscillator, with parameter values $\lambda_{0}=\left(a_{0}, b_{0}\right)=(0.02,3)$ is shown in Fig. 9(a). The two target invariant densities shown in Figs. 9(b) and 9(c) were successfully solved by IFPP and stabilized by parameteric feedback control. The time-dependant parameter controls are shown in Fig. 10, and they are relatively small due to the relatively fine grid. While the supports of the invariant densities shown in Figs. 9(a) and 9(b) appear similar (aside from the removed region), care- ful inspection of the densities reveals that they are usually different.

Two notes of warning are in order. The first is that not all arbitrary densities are reachable with an arbitrarily fine grid. See [Bollt, 2000a] for the controllability theory. The two target densities in Figs. 9(b) and 9(c) represent the two main types of control densities which are exactly attainable. Both these approximate "atypical" invariant densities of the original system. Figure $9(\mathrm{~b})$ shows a nonattracting chaotic saddle, and Fig. 9(c) shows 

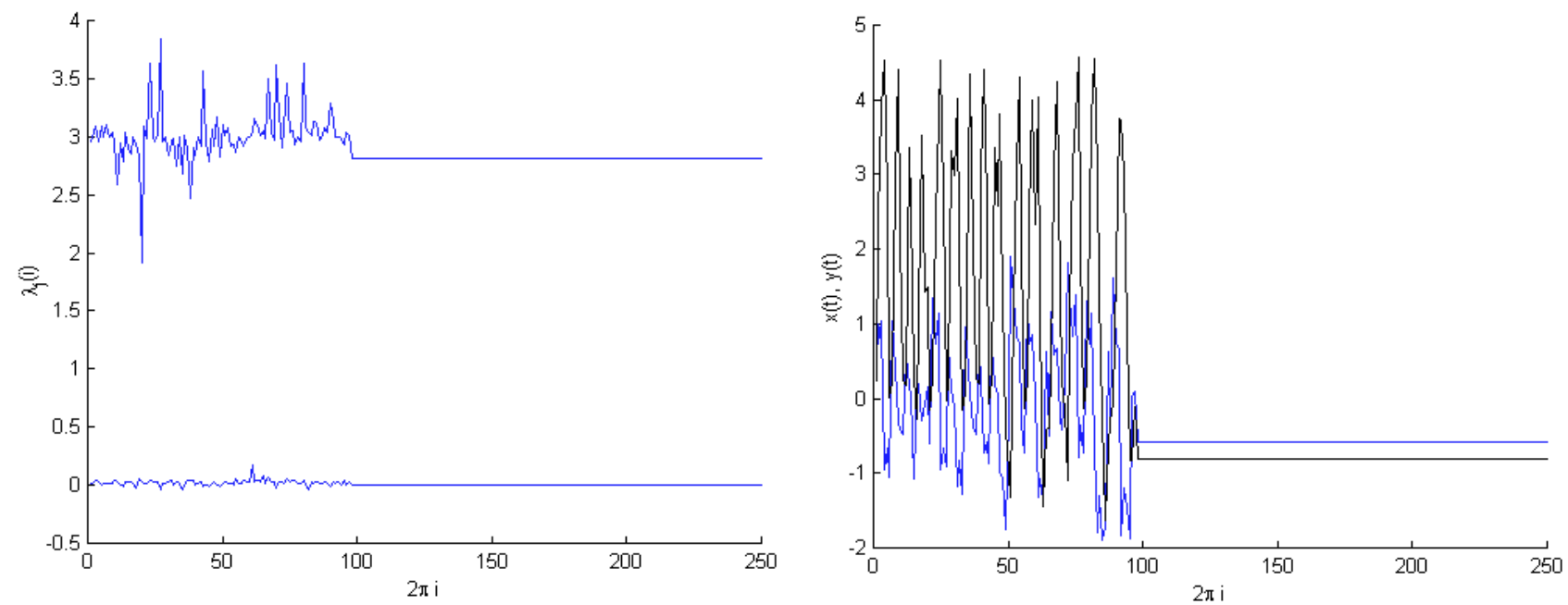

Fig. 10. Time-series of control parameter values (left) and coordinates (right) during feedback control of density shown in Fig. 9(c), globally stabilizing a periodic cycle.

a delta density supported over a periodic orbit. In terms of the graph, these both represent closed subsystems of the original digraph. That is, if one wishes to eliminate a set of vertices from the digraph representation, then the remaining set of vertices must still be closed in that each vertex must have an allowed transition to another remaining vertex, within the original grammar. If such closure is not possible, then a compromise density will automatically be found as the solution of the optimization problem, Eq. (11) subject to constraints Eqs. (8) and (9).

The second note of warning concerns the role of noise and control feedback error when targeting fine-scaled statistical measurements. Obviously fine-scaled structures of the invariant density of a scale smaller than the noise or error are not possible. This is partly, but not fully, solved by our choice of IFS, which allows us to target grid square centers to offer a small amount of noise-immunity. We are currently invesitigating the trade-off between noise, structure scale and attainable density targets.

\section{Conclusion}

In this paper, we have shown that difficult global control problems, which have traditionally been intractable, can be achieved by using a coarsegrained representation of the dynamical system's transfer operators as a directed graph/transfer matrix. Within this formalism, graph theoretic algorithms can be readily posed and applied. In this paper, we have shown that such graph theoretic approaches do indeed apply to the control of a chaotic differential equation, since forcing a true trajectory to follow the trajectory designed on a global scale graph model, is just a matter of continually applying locally calculated parametric feedback control.

In future research, we hope to show that our techniques can be useful in a physical experiment, which will require that we address some of the following fundamental modeling issues. In designing parametric control, it is necessary to be able to predict responses, in order to choose parameter variations. We have taken the point of view, in this proof of principle study, that a good closed form model, in the form of a differential equation, is available to predict next responses due to parameter variations on Poincare' section. This is realistic in the situation that the physics underlying the dynamical process is known and can lead to a differential equation model, in which the associated parameters can often be either directly measured or sometimes inferred. There exist two main approaches to infer, or parameter estimate which we will pursue. The first method is direct calculation. Epureanu and Dowell [1997] linearized around a periodic orbit of the Duffing oscillator, and they derived the sensitivity vector by solving local linear variational equations, by the variation of parameters technique. They used a rather direct method to identify parameters, assuming an ansatz form of the differential equation, $\mathbf{x}^{\prime}=\mathbf{f}(\mathbf{x}, \mathbf{c}, \mathbf{t})$, where $\mathbf{c}$ are the unknown parameters to be fitted. Matching this to $\mathbf{x}^{\prime}=\mathbf{g}\left(\mathbf{x}_{i}, \mathbf{p}_{0}, \mathbf{t}_{i}\right) \approx\left(\mathbf{x}_{i+1}-\mathbf{x}_{i}\right) /\left(\mathbf{t}_{i+1}-\mathbf{t}_{i}\right)$, and using constant time sampled data $\mathbf{x}_{i} \equiv \mathbf{x}\left(\mathbf{t}_{i}\right)$, they 
derived an overdetermined linear system of equations in the unknown parameters c. The second method is called "autosyncronization," [Parlitz, 1996; Parlitz et al., 1996a; Parlitz et al., 1996b], which is closely related to the observer problem from control theory [Rugh, 1996; Antsaklis, 1997; Isidori, 1995]. The idea is that observing a chaotic dynamical system can be achieved by synchronizing a model to a scalar signal from the observed system [Brown \& Bryant, 1991; Brown, 1993]. Parlitz [Parlitz, 1996; Parlitz et al., 1996a; Parlitz et al., 1996b], and similarly Maybhate and Amritkar [1999], used adaptive control based on synchronization to estimate parameters directly from a single scalar time-series. Given a model ansatz, it was shown [Parlitz, 1996; Parlitz et al., 1996a; Parlitz et al., 1996b] that system parameters can be estimated from the sampled scalar time-series data by synchronization between the driving dynamical system generating the data stream and the model augmented by differential equations of the to-be estimated parameters and mediated by synchronization error. On the other hand, we could take the point of view that we wish to control a "black-box" process. Time-series embedding analysis [Takens, 1980] has been shown by several groups [So \& Ott, 1995; Ding et al., 1996; Yang et al., 2000] to be a valid way to sufficiently model to achieve OGYtype control of unstable periodic orbits in a chaotic system which is only known through measurement of a scalar time-series. There is hope that these techniques might be extended to allow for our modeling requirements, but such will surely be data intensive. Hence it is our opinion that whenever a analytic (equations) model is possible, it should be pursued.

\section{Acknowledgments}

The author has been supported by the Naval Academy Research Council (N.A.R.C.), and the National Science Foundation (N.S.F.) under grants DMS-9704639 and DMS-0071314.

\section{References}

Alligood, K. T., Sauer, T. D. \& Yorke, J. A. [1996] Chaos, An Intoduction to Dynamical Systems (Springer, NY).

Antsaklis, P. J. \& Michel, A. N. [1997] Linear Systems (McGraw-Hill).

Artuso, R., Aurell, E. \& Cvitanovic, P. [1990] "Recycling of strange sets II. Applications," Nonlinearity $\mathbf{3}$, p. 361 .
Baranovsky, A. \& Daems, D. [1995] "Design of onedimensional chaotic maps with prescribed statistical properties," Int. J. Bifurcation and Chaos 5(6), 1585-1598.

Barnsley, M. F. [1988] Fractals Everywhere (Academic Press, Boston).

Bollt, E. \& Meiss, J. D. [1995] "Controlling chaos through recurrence," Physica D81, 280-294.

Bollt, E., Lai, Y.-C. \& Grebogi, C. [1997] "Analysis of the topological entropy versus noise resistance tradeoff when communicating with chaos," Phys. Rev. Lett. 79(19), 3787-3790.

Bollt, E. \& Kostelich, E. [1998] "Optimal targeting of chaos," Phys. Lett. A245(5), 399-406.

Bollt, E. [2000a] "Controlling chaos and the inverse Frobenius-Perron problem: Global stabilization of arbitrary invariant measures," Int. J. Bifurication and Chaos 10(5), 1033-1050.

Bollt, E. [2000b] "An $l^{\infty}$ solution to the inverse Frobenius-Perron problem," submitted to ISCAS'2000, Conf. Proc., Geneva, Switzerland, May 2000 available at http://mathweb.mathsci.usna.edu/ faculty/bolltem/.

Bondy, J. A. \& Murty, U. S. R. [1976] Graph Theory with Applications (American Elsevier Publishing Co., NY).

Bowen, R. [1975] Equilibrium States and the Ergodic Theory of Anosov Diffeomorphisms (Springer-Verlag, Berlin).

Boyarsky, A. \& Lou, Y. S. [1991] "Approximating measures invariant under higher-dimensional chaotic transformations," J. Approx. Th. 65, 231-244.

Brown, R. \& Bryant, P. [1991] "Computing the Lyapunov spectrum of a dynamical system from an observed time series," Phys. Rev. A43, 2787-2806.

Brown, R. [1993] "Calculating Lyapunov exponents for short and/or noisy data sets," Phys. Rev. E47, 3962-3969.

Chen, G. \& Lai, D. [1997] "Anticontrol of chaos via feedback," Proc. IEEE Conf. Decis. Contr., San Diego, CA, Dec., 1997, pp. 367-372.

Chen, G. \& Dong, X. [1998] From Chaos to Order, Methodologies, Perspectives and Applications (WorldScientific, Singapore).

Colemann, T., Branch, M. A. \& Grace, A. [1999] Optimization Toolbox, For Use with MatLab,User's Guide, Version 2 (The Math Works Inc., Natick, MA).

Cvitanovic, P., [1988] "Invariant measurements of strange sets in terms of cycles," Phys. Rev. Lett. 61, 2729-2732.

Cvitanovic, P. [1991] "Periodic orbits as the skeleton of classical and quantum chaos," Physica D51 138-151.

Ding, J. \& Zhou, A. H. [1994] "Piecewise linear Markov approximations of Frobenius-Perron operators asscoiated with multi-dimensional transformations," Nonlin. Anal. Th. Methods Appl. 25(4), 399-408. 
Ding, J. \& Zhou, A. H. [1995] "The projection method for computing multi-dimensional absolutely continuous invariant measures," J. Stat. Phys. 77(3 \& 4), 899-908.

Ding, M., Yang, W., In, V., Ditto, W. L., Spano, M. L. \& Gluckman, B. [1996] "Controlling chaos in high dimensions: Theory and experiment," Phys. Rev. E53(5), 4334-4344.

Epureanu, B. I. \& Dowell, E. H. [1997] "System identification for the Ott-Grebogi-Yorke controller design," Phys. Rev. E56(5), 5327-5331.

Fallet, S. [1996] "Algebraic integers and tensor products of matrices," Crux Math. 22, 341-343.

Froyland, G. [1995] "Finite approximation of SinaiBowen-Ruelle measures of Anosov systems in two dimensions," Rand. Comput. Dyn. 3(4), 251-264.

Froyland, G. [1998] "Approximating physical invariant measures of mixing dynamical systems," Nonlin. Anal. 32(7), 831-860.

Góra, P. \& Boyarsky, A. [1993] "A matrix solution to the inverse Frobenius-Perron problem," Proc. AMS 118(2), 409-414.

Góra, P. \& Boyarsky, A. [1996] "An algorithm to control chaotic behavior in one-dimensional maps," Comp. Math. Appl. 31(6), 13-22.

Góra, P. \& Boyarsky, A. [1997] Laws of Chaos, Invariant Measures and Dynamical Systems in One Dimension (Birkhäuser, Boston).

Góra, P. \& Boyarsky, A. [1998] "A new approach to controlling chaotic systems," Physica D111, 1-15.

Góra, P. \& Boyarsky, A. [1999] "Optimal control of chaotic systems," Int. J. Bifurcation and Chaos, to appear.

Gould, R. [1988] Graph Theory (Benjamin/Cummings Publishing Co., Inc., Menlo Park, CA).

Isidori, A. [1995] Nonlinear Control Systems, 3rd edition (Springer-Verlag).

Kadanoff, L. P. \& Tang, C. [1984] "Escape from strange repellers," Proc. Natl. Acad. Sci, USA 81, 1276-1279.

Koga, S. [1991] "The inverse problem of FrobeniusPerron equations in 1D difference systems," Progr. Theor. Phys. 86(5), 991-1002.

Kostelich, E. J., Grebogi, C., Ott, E. \& Yorke, E. [1993] "Higher dimensional targeting," Phys. Rev. E47, 305-310.

Lasota, A. \& Mackey, M. [1997] Chaos, Fractals, and Noise, 2nd edition (Springer-Verlag, NY).

Li, T.-Y. [1976] "Finite approximation for the FrobeniusPerron operator. A solution to Ulam's conjecture," $J$. Approx. Th. 17, 177-186.

Lorenz, E. [1963] "Deterministic nonperiodic flow," $J$. Atm. Sci. 20, 130-141.

Maybhate, A. \& Amritkar, R. E. [1999] "Use of synchronization and adaptive control in parameter estimation from a time series," Phys. Rev. E59, 284-293.
Ott, E., Grebogi, C. \& Yorke, J. A. [1990] "Controlling chaos," Phys. Rev. Lett. 64, 1196-1199.

Ott, E., Sauer, T. \& Yorke, J. A. [1994] Coping with Chaos: Analysis of Chaotic Data and The Exploitation of Chaotic Systems (Wiley-Interscience).

Parlitz, U. [1996] "Estimating model parameters from time series by autosynchronization," Phys. Rev. Lett. 76, 1232-1235.

Parlitz, U., Kocarev, L., Stojanovski, T. \& Preckel, H. [1996a] Phys. Rev. E53, 4351-4361.

Parlitz, U., Junge, L. \& Kocarev, L. [1996b] Phys. Rev. E54, 6253-6259.

Pecora, L. M., Carroll, T. L., Johnson, G. A. \& Mar, D. J. [1997] "Fundamentals of synchronization in chaotic systems, concepts, and applications," Chaos 7(4), 520-543.

Pecora, L. M. \& Carroll, T. L. [1998] "Master stability functions for syncronizated coupled systems," Phys. Rev. Lett. 80, 2729-2732.

Pingel, D., Schmelcher, P. \& Diakonos, F. K. [1999] "Theory and examples of the inverse-FrobeniusPerron problem for complete chaotic maps," Chaos 9, 357-366.

Press, W. H., Flannery, B. P., Teukolovsky, S. A. \& Vetterling, W. T. [1986] Numerical Recipes (Cambridge University Press, Cambridge).

Proppe, H., Góra, P. \& Boyarsky, A. [1990] "Indadequacy of the bounded variation technique in the ergodic theory of higher-dimensional transformations," Nonlinearity 3, 1081-1087.

Rugh, W. J. [1996] Linear Systems Theory, 2nd edition (Prentice Hall).

Schiff, S. J., Jerger, K., Duong, D. H., Chang, T., Spano, M. L. \& Ditto, W. L. [1994] "Controlling chaos in the brain," Nature 370, 615-620.

Shinbrot, T., Ott, E., Grebogi, C. \& Yorke, J. A. [1990] "Using chaos to direct trajectories to targets," Phys. Rev. Lett. 65, 3215-3218.

Shinbrot, T., Ditto, W., Grebogi, C., Ott, E., Spano, M. \& Yorke, J. A. [1992] "Using the sensitive dependence of chaos (the "butterfly effect") to direct trajectories in an experimental chaotic system," Phys. Rev. Lett. 68(19), 2863-2866.

So, P. \& Ott, E. [1995] "Controlling chaos using time delay coordinates via stabilization of periodic orbits," Phys. Rev. E51(4), 2955-2962.

Takens, F. [1980] "Detecting strange attractors in turbulence," in Dynamical Systems and Turbulence, eds. Rand, D. A. \& Young, L.-S., Lecture Notes in Mathematics, Vol. 898 (Springer-Verlag, Berlin), pp. 336-381.

Ulam, S. [1960] Problems in Modern Mathematics (Interscience Publishers, NY).

Yang, L., Liu, Z. \& Mao, J.-M. [2000] "Controlling hyperchaos," Phys. Rev. Lett. 84, 67-70. 